\title{
Constructing Lefschetz fibrations via daisy substitutions
}

\author{
Anar Akhmedov and Naoyuki Monden
}

\begin{abstract}
We construct new families of nonhyperelliptic Lefschetz fibrations by applying the daisy substitutions to the families of words $\left(c_{1} c_{2} \cdots c_{2 g-1} c_{2 g} c_{2 g+1}{ }^{2} c_{2 g}\right.$. $\left.c_{2 g-1} \cdots c_{2} c_{1}\right)^{2}=1,\left(c_{1} c_{2} \cdots c_{2 g} c_{2 g+1}\right)^{2 g+2}=1$, and $\left(c_{1} c_{2} \cdots c_{2 g-1} c_{2 g}\right)^{2(2 g+1)}=1$ in the mapping class group $\Gamma_{g}$ of the closed orientable surface of genus $g$, and we study the sections of these Lefschetz fibrations. Furthermore, we show that the total spaces of some of these Lefschetz fibrations are irreducible exotic 4-manifolds, and we compute their Seiberg-Witten invariants. By applying the knot surgery to the family of Lefschetz fibrations obtained from the word $\left(c_{1} c_{2} \cdots c_{2 g} c_{2 g+1}\right)^{2 g+2}=1$ via daisy substitutions, we also construct an infinite family of pairwise nondiffeomorphic irreducible symplectic and nonsymplectic 4-manifolds homeomorphic to $\left(g^{2}-g+1\right) \mathbb{C P}^{2} \#\left(3 g^{2}-g(k-3)+\right.$ $2 k+3) \overline{\mathbb{C P}}^{2}$ for any $g \geq 3$ and $k=2, \ldots, g+1$.
\end{abstract}

\section{Introduction}

The Lefschetz fibrations are fundamental objects to study in 4-dimensional topology. In his remarkable works, Simon Donaldson [9] showed that every closed symplectic 4-manifold admits a structure of Lefschetz pencil, which can be blown up at its base points to yield a Lefschetz fibration, and conversely, Robert Gompf [19] showed that the total space of a genus $g$ Lefschetz fibration admits a symplectic structure, provided that the homology class of the fiber is nontrivial. Given a Lefschetz fibration over $\mathbb{S}^{2}$, one can associate to it a word in the mapping class group of the fiber composed solely of right-handed Dehn twists, and conversely, given such a factorization in the mapping class group, one can construct a Lefschetz fibration over $\mathbb{S}^{2}$ (see, e.g., [19]).

Recently, there has been much interest in trying to understand the topological interpretation of various relations in the mapping class group. A particularly well-understood case is the daisy relation, which corresponds to the symplectic operation of rational blowdown (see [11], [12]). Another interesting problem, which is still open, is whether any Lefschetz fibration over $\mathbb{S}^{2}$ admits a section

Kyoto Journal of Mathematics, Vol. 56, No. 3 (2016), 501-529

DOI $10.1215 / 21562261-3600148$, C 2016 by Kyoto University

Received July 1, 2014. Revised March 9, 2015. Accepted May 13, 2015.

2010 Mathematics Subject Classification: Primary 57R55; Secondary 57R17.

Akhmedov's work partially supported by National Science Foundation grants FRG-1065955 and DMS-1005741 and a Sloan Fellowship.

Monden's work partially supported by Grant-in-Aid for Young Scientists (B) (No. 13276356), Japan Society for the Promotion of Science. 
(see, e.g., [33]). Furthermore, one would like to determine how many disjoint sections the given Lefschetz fibration admits. The later problem has been studied for the standard family of hyperelliptic Lefschetz fibrations (with total spaces $\left.\mathbb{C P}^{2} \#(4 g+5) \overline{\mathbb{C P}}^{2}\right)$ in [25], [28], and [35], using the computations in the mapping class group, and such results are useful in constructing (exotic) Stein fillings (see [1], [2]).

Motivated by these results and problems, our goal in this article is to construct new families of Lefschetz fibrations over $\mathbb{S}^{2}$ by applying the sequence of daisy substitutions and conjugations to the hyperelliptic words $\left(c_{1} c_{2} \cdots c_{2 g-1}\right.$. $\left.c_{2 g} c_{2 g+1}^{2} c_{2 g} c_{2 g-1} \cdots c_{2} c_{1}\right)^{2}=1,\left(c_{1} c_{2} \cdots c_{2 g} c_{2 g+1}\right)^{2 g+2}=1$, and $\left(c_{1} c_{2} \cdots c_{2 g-1}\right.$. $\left.c_{2 g}\right)^{2(2 g+1)}=1$ in the mapping class group of the closed orientable surface of genus $g$ for any $g \geq 3$ and study the sections of these Lefschetz fibrations (cf. Theorems 24-28). Furthermore, we show that the total spaces of our Lefschetz fibrations given by the last two words are irreducible exotic symplectic 4-manifolds, and we compute their Seiberg-Witten invariants (cf. Theorem 31). Analogous (but weaker) results for the special case of $g=2$, using the lantern substitutions only, were obtained in [11] and [3]. We would like to remark that the mapping class group computations in our article are more involved and subtle than those in [1] and [3]. One family of examples, obtained from the fiber sums of the Lefschetz fibrations by using daisy relations, were studied in [12]. However, the examples obtained in [12] have larger topology, and the computations of Seiberg-Witten invariants and study of sections were not addressed in [12]. Moreover, we prove the nonhyperellipticity of our Lefschetz fibrations and provide some criteria for nonhyperellipticity under the daisy substitutions (cf. Theorem 29). Some of our examples can be used to produce the families of nonisomorphic Lefschetz fibrations over $\mathbb{S}^{2}$ with the same total spaces and exotic Stein fillings. We hope to return to these examples in future work.

The organization of our article is as follows. In Sections 2 and 3 we recall the main definitions and results that will be used throughout the article. In Section 4, we prove some technical lemmas, important in the proofs of our main theorems. In Sections 5 and 6, we construct new families of Lefschetz fibrations by applying the daisy substitutions to the words given above, study the sections, and prove the nonhyperellipticity of our Lefschetz fibrations (Theorems 24-29). Finally, in Section 7, we prove that the total spaces of some of these Lefschetz fibrations are exotic symplectic 4-manifolds, which we verify by computing their Seiberg-Witten invariants and obtaining an infinite family of exotic 4-manifolds via knot surgery (Theorems 31,32), and make some remarks and raise questions. We would like to remark that the main technical content of our article is more algebraic since our proofs rely heavily on mapping class group techniques. It is possible to pursue a more geometric approach (see Example 12), but such an approach alone does not yield the optimal results as presented here. 


\section{Mapping class groups}

Let $\Sigma_{g}^{n}$ be a 2-dimensional, compact, oriented, and connected surface of genus $g$ with $n$ boundary components. Let $\operatorname{Diff}^{+}\left(\Sigma_{g}^{n}\right)$ be the group of all orientationpreserving self-diffeomorphisms of $\Sigma_{g}^{n}$ which are the identity on the boundary, and let $\operatorname{Diff}_{0}^{+}\left(\Sigma_{g}\right)$ be the subgroup of $\operatorname{Diff}^{+}\left(\Sigma_{g}\right)$ consisting of all orientationpreserving self-diffeomorphisms that are isotopic to the identity. The isotopies are also assumed to fix the points on the boundary. The mapping class group $\Gamma_{g}^{n}$ of $\Sigma_{g}^{n}$ is defined to be the group of isotopy classes of orientation-preserving diffeomorphisms of $\Sigma_{g}^{n}$; that is,

$$
\Gamma_{g}^{n}=\operatorname{Diff}^{+}\left(\Sigma_{g}^{n}\right) / \operatorname{Diff}_{0}^{+}\left(\Sigma_{g}^{n}\right) .
$$

For simplicity, we write $\Sigma_{g}=\Sigma_{g}^{0}$ and $\Gamma_{g}=\Gamma_{g}^{0}$. The hyperelliptic mapping class group $H_{g}$ of $\Sigma_{g}$ is defined as the subgroup of $\Gamma_{g}$ consisting of all isotopy classes commuting with the isotopy class of the hyperelliptic involution $\iota: \Sigma_{g} \rightarrow \Sigma_{g}$.

\section{DEFINITION 1}

Let $\alpha$ be a simple closed curve on $\Sigma_{g}^{n}$. A right-handed (or positive) Dehn twist about $\alpha$ is a diffeomorphism of $t_{\alpha}: \Sigma_{g}^{n} \rightarrow \Sigma_{g}^{n}$ obtained by cutting the surface $\Sigma_{g}^{n}$ along $\alpha$ and gluing the ends back after rotating one of the ends $2 \pi$ to the right.

It is well known that the mapping class group $\Gamma_{g}^{n}$ is generated by Dehn twists. It is an elementary fact that the conjugate of a Dehn twist is again a Dehn twist: if $\phi: \Sigma_{g}^{n} \rightarrow \Sigma_{g}^{n}$ is an orientation-preserving diffeomorphism, then $\phi \circ t_{\alpha} \circ \phi^{-1}=t_{\phi(\alpha)}$. The following lemma is easy to verify (see [22] for a proof).

\section{LEMMA 2}

Let $\alpha$ and $\beta$ be two simple closed curves on $\Sigma_{g}^{n}$. If $\alpha$ and $\beta$ are disjoint, then their corresponding Dehn twists satisfy the commutativity relation $t_{\alpha} t_{\beta}=t_{\beta} t_{\alpha}$. If $\alpha$ and $\beta$ transversely intersect at a single point, then their corresponding Dehn twists satisfy the braid relation $t_{\alpha} t_{\beta} t_{\alpha}=t_{\beta} t_{\alpha} t_{\beta}$.

\subsection{Daisy relation and daisy substitution}

We recall the definition of the daisy relation (see [30], [12], [7]).

\section{DEFINITION 3}

Let $\Sigma_{0}^{p+2}$ denote a sphere with $p+2$ boundary components $(p \geq 2)$. Let $\delta_{0}, \delta_{1}, \delta_{2}$, $\ldots, \delta_{p+1}$ be the $p$ boundary curves of $\Sigma_{0}^{p+2}$, and let $x_{1}, x_{2}, \ldots, x_{p+1}$ be the interior curves as shown in Figure 1. Then, we have the daisy relation of type $p$

$$
t_{\delta_{0}}^{p-1} t_{\delta_{1}} t_{\delta_{2}} \cdots t_{\delta_{p+1}}=t_{x_{1}} t_{x_{2}} \cdots t_{x_{p+1}} .
$$

We call the following relator the daisy relator of type $p$ :

$$
t_{\delta_{p+1}}^{-1} \cdots t_{\delta_{2}}^{-1} t_{\delta_{1}}^{-1} t_{\delta_{0}}^{-p+1} t_{x_{1}} t_{x_{2}} \cdots t_{x_{p+1}}(=1) .
$$




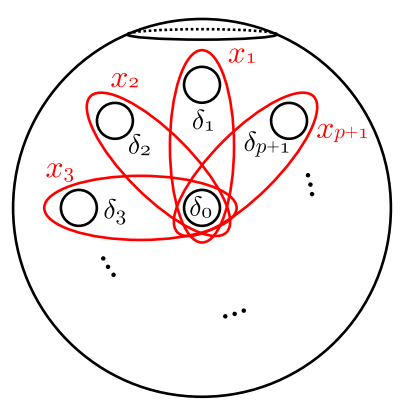

Figure 1. Daisy relation.

\section{REMARK 4}

When $p=2$, the daisy relation is commonly known as the lantern relation (see [8], [23]).

We next introduce a daisy substitution, a substitution technique introduced by T. Fuller.

\section{DEFINITION 5}

Let $d_{1}, \ldots, d_{m}$ and $e_{1}, \ldots, e_{n}$ be simple closed curves on $\Gamma_{g}^{n}$, and let $R$ be a product $R=t_{d_{1}} t_{d_{2}} \cdots t_{d_{l}} t_{e_{m}}^{-1} \cdots t_{e_{2}}^{-1} t_{e_{1}}^{-1}$. Suppose that $R=1$ in $\Gamma_{g}^{n}$. Let $\varrho$ be a word in $\Gamma_{g}^{n}$ including $t_{d_{1}} t_{d_{2}} \cdots t_{d_{l}}$ as a subword:

$$
\varrho=U \cdot t_{d_{1}} t_{d_{2}} \cdots t_{d_{l}} \cdot V
$$

where $U$ and $V$ are words. Thus, we obtain a new word in $\Gamma_{g}^{n}$, denoted by $\varrho^{\prime}$, as follows:

$$
\varrho^{\prime}:=U \cdot t_{e_{1}} t_{e_{2}} \cdots t_{e_{m}} \cdot V
$$

Then, we say that $\varrho^{\prime}$ is obtained by applying an $R$-substitution to $\varrho$. In particular, if $R$ is a daisy relator of type $p$, then we say that $\varrho^{\prime}$ is obtained by applying a daisy substitution of type $p$ to $\varrho$.

\section{Lefschetz fibrations}

\section{DEFINITION 6}

Let $X$ be a closed, oriented smooth 4-manifold. A smooth map $f: X \rightarrow \mathbb{S}^{2}$ is a genus $g$ Lefschetz fibration if it satisfies the following conditions:

(i) $f$ has finitely many critical values $b_{1}, \ldots, b_{m} \in S^{2}$, and $f$ is a smooth $\Sigma_{g}$-bundle over $\mathbb{S}^{2}-\left\{b_{1}, \ldots, b_{m}\right\}$;

(ii) for each $i(i=1, \ldots, m)$, there exists a unique critical point $p_{i}$ in the singular fiber $f^{-1}\left(b_{i}\right)$ such that about each $p_{i}$ and $b_{i}$ there are local complex coordinate charts agreeing with the orientations of $X$ and $\mathbb{S}^{2}$ on which $f$ is of the form $f\left(z_{1}, z_{2}\right)=z_{1}^{2}+z_{2}^{2}$;

(iii) $f$ is relatively minimal (i.e., no fiber contains a (-1)-sphere). 
Each singular fiber is obtained by collapsing a simple closed curve (the vanishing cycle) in the regular fiber. The monodromy of the fibration around a singular fiber is given by a right-handed Dehn twist along the corresponding vanishing cycle. For a genus $g$ Lefschetz fibration over $\mathbb{S}^{2}$, the product of right-handed Dehn twists $t_{v_{i}}$ about the vanishing cycles $v_{i}$, for $i=1, \ldots, m$, gives us the global monodromy of the Lefschetz fibration, the relation $t_{v_{1}} t_{v_{2}} \cdots t_{v_{m}}=1$ in $\Gamma_{g}$. This relation is called the positive relator. Conversely, such a positive relator defines a genus $g$ Lefschetz fibration over $\mathbb{S}^{2}$ with the vanishing cycles $v_{1}, \ldots, v_{m}$.

According to Kas [24] and Matsumoto [27], if $g \geq 2$, then the isomorphism class of a Lefschetz fibration is determined by a positive relator modulo simultaneous conjugations

$$
t_{v_{1}} t_{v_{2}} \cdots t_{v_{m}} \sim t_{\phi\left(v_{1}\right)} t_{\phi\left(v_{2}\right)} \cdots t_{\phi\left(v_{m}\right)} \quad \text { for all } \phi \in M_{g}
$$

and elementary transformations

$$
\begin{aligned}
& t_{v_{1}} \cdots t_{v_{i-1}} t_{v_{i}} t_{v_{i+1}} t_{v_{i+2}} \cdots t_{v_{m}} \sim t_{v_{1}} \cdots t_{v_{i-1}} t_{t_{v_{i}}\left(v_{i+1}\right)} t_{v_{i}} t_{v_{i+2}} \cdots t_{v_{m}} \\
& t_{v_{1}} \cdots t_{v_{i-2}} t_{v_{i-1}} t_{v_{i}} t_{v_{i+1}} \cdots t_{v_{m}} \sim t_{v_{1}} \cdots t_{v_{i-2}} t_{v_{i}} t_{t_{v_{i}}^{-1}\left(v_{i-1}\right)} t_{v_{i+1}} \cdots t_{v_{m}} .
\end{aligned}
$$

Note that $\phi t_{v_{i}} \phi^{-1}=t_{\phi\left(v_{i}\right)}$. We denote a Lefschetz fibration associated to a positive relator $\varrho \in \Gamma_{g}$ by $f_{\varrho}$.

For a Lefschetz fibration $f: X \rightarrow \mathbb{S}^{2}$, a map $\sigma: \mathbb{S}^{2} \rightarrow X$ is called a section of $f$ if $f \circ \sigma=\mathrm{id}_{\mathbb{S}^{2}}$. We define the self-intersection of $\sigma$ to be the self-intersection number of the homology class $\left[\sigma\left(\mathbb{S}^{2}\right)\right]$ in $H_{2}(X ; \mathbb{Z})$. Let $\delta_{1}, \delta_{2}, \ldots, \delta_{n}$ be $n$ boundary curves of $\Sigma_{g}^{n}$. If there exists a lift of a positive relator $\varrho=t_{v_{1}} t_{v_{2}} \cdots t_{v_{m}}=1$ in $\Gamma_{g}$ to $\Gamma_{g}^{n}$ as

$$
t_{\tilde{v}_{1}} t_{\tilde{v}_{2}} \cdots t_{\tilde{v}_{m}}=t_{\delta_{1}} t_{\delta_{2}} \cdots t_{\delta_{n}}
$$

then $f_{\varrho}$ admits $n$ disjoint sections of self-intersection -1 . Here, $t_{\tilde{v}_{i}}$ is a Dehn twist mapped to $t_{v_{i}}$ under $\Gamma_{g}^{n} \rightarrow \Gamma_{g}$. Conversely, if a genus $g$ Lefschetz fibration admits $n$ disjoint sections of self-intersection -1 , then we obtain such a relation in $\Gamma_{g}^{n}$.

Next, let us recall the signature formula for hyperelliptic Lefschetz fibrations, which is due to Matsumoto and Endo. We will make use of this formula in Section 6, where we prove that all our Lefschetz fibrations obtained via daisy substitutions are nonhyperelliptic.

\section{THEOREM 7 ([26], [27], [10])}

Let $f: X \rightarrow \mathbb{S}^{2}$ be a genus $g$ hyperelliptic Lefschetz fibration. Let $s_{0}$ and $s=$ $\sum_{h=1}^{[g / 2]} s_{h}$ be the number of nonseparating and separating vanishing cycles of $f$, where $s_{h}$ denotes the number of separating vanishing cycles which separate the surface of genus $g$ into two surfaces, one of which has genus $h$. Then, we have the following formula for the signature:

$$
\sigma(X)=-\frac{g+1}{2 g+1} s_{0}+\sum_{h=1}^{\left[\frac{g}{2}\right]}\left(\frac{4 h(g-h)}{2 g+1}-1\right) s_{h} .
$$




\subsection{Spinness criteria for Lefschetz fibrations}

In this section, we recall a few results due to A. Stipsicz [34] concerning the nonspinness and spinness of the Lefschetz fibrations over $\mathbb{D}^{2}$ and $\mathbb{S}^{2}$. We will use them to verify that our families of Lefschetz fibrations in Theorem 31 all are nonspin. Since Rohlin's criteria [31] cannot be used to verify nonspinness when the signature of our families of Lefschetz fibrations is divisible by 16, Stipsicz's results will be more suitable for our purpose.

Let $f: X \rightarrow \mathbb{D}^{2}$ be a Lefschetz fibration over a disk, and let $F$ denote the generic fiber of $f$. Denote the homology classes of the vanishing cycles of the given fibration by $v_{1}, \ldots, v_{m} \in H_{1}\left(F ; \mathbb{Z}_{2}\right)$.

\section{THEOREM 8 ([34])}

The Lefschetz fibration $f: X \rightarrow \mathbb{D}^{2}$ is not spin if and only if there are $l$ vanishing cycles $v_{1}, \ldots, v_{l}$ such that $v=\sum_{i=1}^{l} v_{i}$ is also a vanishing cycle and $l+$ $\sum_{1 \leq i<j \leq l} v_{i} \cdot v_{j} \equiv 0(\bmod 2)$.

Note that the above theorem implies that if the Lefschetz fibration has the separating vanishing cycle, then its total space is not spin. To see this, set $l=0$, and take the empty sum to be 0 .

\section{THEOREM 9 ([34])}

The Lefschetz fibration $f: X \rightarrow \mathbb{S}^{2}$ is spin if and only if $X \backslash \nu(F)$ is spin and for some dual $\sigma$ of $F$ we have $\sigma^{2} \equiv 0(\bmod 2)$.

\subsection{Three families of hyperelliptic Lefschetz fibrations}

In this section, we introduce three well-known families of hyperelliptic Lefschetz fibrations, which will serve as building blocks in our construction of new Lefschetz fibrations. Let $c_{1}, c_{2}, \ldots, c_{2 g}, c_{2 g+1}$ denote the collection of simple closed curves given in Figure 2, and let $c_{i}$ denote the right-handed Dehn twists $t_{c_{i}}$ along the curve $c_{i}$. It is well known that the following relations hold in the mapping class group $\Gamma_{g}$ :

$$
\begin{aligned}
H(g) & =\left(c_{1} c_{2} \cdots c_{2 g-1} c_{2 g} c_{2 g+1}{ }^{2} c_{2 g} c_{2 g-1} \cdots c_{2} c_{1}\right)^{2}=1, \\
I(g) & =\left(c_{1} c_{2} \cdots c_{2 g} c_{2 g+1}\right)^{2 g+2}=1, \\
G(g) & =\left(c_{1} c_{2} \cdots c_{2 g-1} c_{2 g}\right)^{2(2 g+1)}=1 .
\end{aligned}
$$

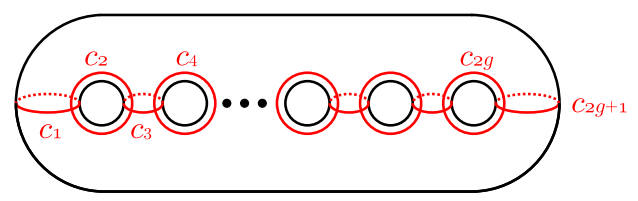

Figure 2. Vanishing cycles of the genus $g$ Lefschetz fibration on $X(g), Y(g)$, and $Z(g)$. 
Let $X(g), Y(g)$, and $Z(g)$ denote the total spaces of the above genus $g$ hyperelliptic Lefschetz fibrations given by the monodromies $H(g)=1, I(g)=1$, and $J(g)=1$, respectively, in the mapping class group $\Gamma_{g}$. For the first monodromy relation, the corresponding genus $g$ Lefschetz fibrations over $\mathbb{S}^{2}$ have total space $X(g)=\mathbb{C P}^{2} \#(4 g+5) \overline{\mathbb{C P}}^{2}$, the complex projective plane blown up at $4 g+5$ points. In the case of the second and third relations, the total spaces of the corresponding genus $g$ Lefschetz fibrations over $\mathbb{S}^{2}$ are also well-known families of complex surfaces. For example, $Y(2)=K 3 \# 2 \overline{\mathbb{C P}}^{2}$ and $Z(2)$ is the Horikawa surface. In what follows, we recall the branched-cover description of the 4-manifolds $Y(g)$ and $Z(g)$, which we will use in the proofs of our main results. The branched-cover description of $X(g)$ is well known, and we refer the reader to [19, Remark 7.3 .5 , p. 257].

\section{LEMMA 10}

The genus $g$ Lefschetz fibration on $Y(g)$ over $\mathbb{S}^{2}$ with the monodromy $\left(c_{1} c_{2} \ldots\right.$ $\left.c_{2 g+1}\right)^{2 g+2}=1$ can be obtained as the double branched covering of $\mathbb{C P}^{2} \# \overline{\mathbb{C P}}^{2}$ branched along a smooth algebraic curve $B$ in the linear system $|2(g+1) \tilde{L}|$, where $\tilde{L}$ is the proper transform of line $L$ in $\mathbb{C P}^{2}$ avoiding the blown-up point. Furthermore, this Lefschetz fibration admits two disjoint (-1)-sphere sections.

\section{Proof}

We will follow the proof of [4, Lemma 3.1], where the $g=2$ case has been considered (see also the discussion in [6]), and make necessary adjustments where needed. Let $D$ denote an algebraic curve of degree $d$ in $\mathbb{C P}^{2}$. We fix a generic projection map $\pi: \mathbb{C P}^{2} \backslash p t \rightarrow \mathbb{C P}^{1}$ such that the pole of $\pi$ does not belong to $D$. It was shown in [37] that the braid monodromy of $D$ in $\mathbb{C P}^{2}$ is given via a braid factorization. More specifically, the braid monodromy around the point at infinity in $\mathbb{C P}^{1}$, which is given by the central element $\Delta^{2}$ in $B_{d}$, can be written as the product of the monodromies about the critical points of $\pi$. Hence, the factorization $\Delta^{2}=\left(\sigma_{1} \cdots \sigma_{d-1}\right)^{d}$ holds in the braid group $B_{d}$, where $\sigma_{i}$ denotes a positive half-twist exchanging two points and fixing the remaining $d-2$ points.

Now let us degenerate the smooth algebraic curve $B$ in $\mathbb{C P}^{2} \# \overline{\mathbb{C P}}^{2}$ into a union of $2(g+1)$ lines in a general position. By the discussion above, the braid group factorization corresponding to the configuration $B$ is given by $\Delta^{2}=$ $\left(\sigma_{1} \sigma_{2} \cdots \sigma_{2 g} \sigma_{2 g+1}\right)^{2 g+2}$. Now, by lifting this braid factorization to the mapping class group of the genus $g$ surface, we obtain the monodromy factorization $\left(c_{1} c_{2} \cdots c_{2 g+1}\right)^{2 g+2}=1$ for the corresponding double branched covering.

Moreover, observe that a regular fiber of the given fibration is a 2 -fold cover of a sphere in $\mathbb{C P}^{2} \# \overline{\mathbb{C P}}^{2}$ with homology class $f=h-e_{1}$ branched over $2(g+1)$ points, where $h$ denotes the hyperplane class in $\mathbb{C P}^{2}$. Hence, a regular fiber is a surface of genus $g$. The exceptional sphere $e_{1}$ in $\mathbb{C P}^{2} \# \overline{\mathbb{C P}}^{2}$, which intersects $f=h-e_{1}$ once positively, lifts to two disjoint $(-1)$-sphere sections in $Y(g)$. 
The proof of the following lemma can be extracted from [19, Example 7.3.27, p. 268]; we omit the proof.

\section{LEMMA 11}

The double branched cover $W(g)$ of $\mathbb{C P}^{2}$ along a smooth algebraic curve $B$ in the linear system $|2(g+1) \tilde{L}|$ can be decomposed as the fiber sum of two copies of $\mathbb{C P}^{2} \#(g+1)^{2} \overline{\mathbb{C P}}^{2}$ along a complex curve of genus equal to $g(g-1) / 2$. Moreover, $W(g)$ admits a genus $g$ Lefschetz pencil with two base points, and $Y(g)=$ $W(g) \# 2 \overline{\mathbb{C P}}^{2}$.

\section{EXAMPLE 12}

In this example, we study the topology of complex surfaces $W(g)$ in some detail. Recall that by Lemma 11 the complex surface $W(g)$ is the fiber sum of two copies of the rational surface $\mathbb{C P}^{2} \#\left(g^{2}+2 g+1\right) \overline{\mathbb{C P}}^{2}$ along the complex curve $\Sigma$ of genus $g(g-1) / 2$ and self-intersection zero. Using the fiber sum decomposition, we compute the Euler characteristic and the signature of $W(g)$ as follows: $e(W(g))=$ $2 e\left(\mathbb{C P}^{2} \#\left(g^{2}+2 g+1\right) \overline{\mathbb{C P}}^{2}\right)-2 e(\Sigma)=4 g^{2}+2 g+4$, and $\sigma(W(g))=2 \sigma\left(\mathbb{C P}^{2} \#\left(g^{2}+\right.\right.$ $\left.2 g+1) \overline{\mathbb{C P}}^{2}\right)=-2\left(g^{2}+2 g\right)$. Next, we recall from [17] that $\mathbb{C P}^{2} \#\left(g^{2}+2 g+1\right) \overline{\mathbb{C P}}^{2}=$ $\Phi_{g(g-1) / 2}(1) \cup N_{g(g-1) / 2}(1)$, where $\Phi_{g(g-1) / 2}(1)$ and $N_{g(g-1) / 2}(1)$ are the Milnor fiber and generalized Gompf nucleus in $\mathbb{C P}^{2} \#\left(g^{2}+2 g+1\right) \overline{\mathbb{C P}}^{2}$, respectively. Notice that this decomposition shows that the intersection form of $\mathbb{C P}^{2} \#\left(g^{2}+\right.$ $2 g+1) \overline{\mathbb{C P}}^{2}$ splits as $N \oplus M(g)$, where $N=\left(\begin{array}{cc}0 & 1 \\ 1 & -1\end{array}\right)$ and $M(g)$ is a matrix whose entries are given by a negative definite plumbing tree in Figure 3. Consequently, we obtain the following decomposition of the intersection form of $W(g): 2 M(g) \oplus$ $H \oplus g(g-1) H$, where $H$ is a hyperbolic pair. Let us choose the following basis which realizes the intersection matrix $M(g) \oplus N$ of $\mathbb{C P}^{2} \#\left(g^{2}+2 g+1\right) \overline{\mathbb{C P}}^{2}:\langle f=$ $(g+1) h-e_{1}-\cdots-e_{(g+1)^{2}}, e_{(g+1)^{2}}, e_{1}-e_{2}, e_{2}-e_{3}, \ldots, e_{(g+1)^{2}-2}-e_{(g+1)^{2}-1}, h-$ $\left.e_{(g+1)^{2}-(g+1)}-\cdots-e_{(g+1)^{2}-2}-e_{(g+1)^{2}-1}\right\rangle$. Observe that the last $(g+1)^{2}-1$ classes can be represented by spheres and their self-intersections are given as in Figure 3. Here, $f$ is the class of fibers of the genus $g(g-1) / 2$ Lefschetz fibration on $\mathbb{C P}^{2} \#\left(g^{2}+2 g+1\right) \overline{\mathbb{C P}}^{2}$ and $e_{(g+1)^{2}}$ is a sphere section of self-intersection -1 . By using the generalized fiber sum decomposition of $W(g)$, it is not hard to see the surfaces that generate the intersection matrix $2 M(g) \oplus H \oplus g(g-1) H$. The two copies of the Milnor fiber $\Phi_{g(g-1) / 2}(1) \subset \mathbb{C P}^{2} \#\left(g^{2}+2 g+1\right) \overline{\mathbb{C P}}^{2}$ are in $W(g)$, providing $2\left((g+1)^{2}-1\right)$ spheres of self-intersections -2 and $-g$ (corresponding to the classes $\left\{e_{1}-e_{2}, e_{2}-e_{3}, \ldots, e_{(g+1)^{2}-2}-e_{(g+1)^{2}-1}, h-e_{(g+1)^{2}-(g+1)}-\cdots-\right.$

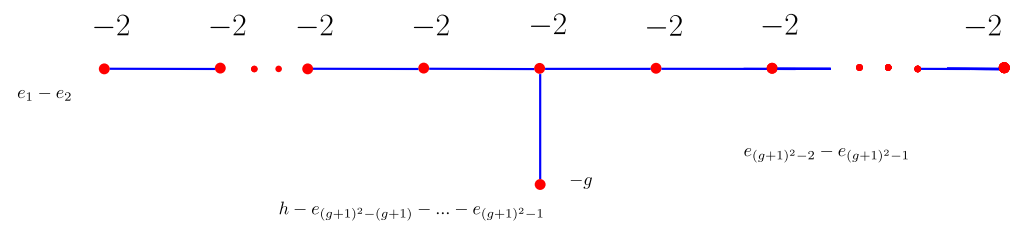

Figure 3. Plumbing tree for $\Phi_{g(g-1) / 2}(1)$. 
$\left.e_{(g+1)^{2}-3}+e_{(g+1)^{2}-2}+e_{(g+1)^{2}-1}\right\}$ and $\left\{e_{1}^{\prime}-e_{2}^{\prime}, e_{2}^{\prime}-e_{3}^{\prime}, \ldots, e_{(g+1)^{2}-2}^{\prime}-e_{(g+1)^{2}-1}^{\prime}\right.$, $\left.\left.h^{\prime}-e_{(g+1)^{2}-(g+1)}-\cdots-e_{(g+1)^{2}-3}^{\prime}-e_{(g+1)^{2}-2}^{\prime}-e_{(g+1)^{2}-1}^{\prime}\right\}\right)$, and realize two copies of $M(g)$. One copy of the hyperbolic pair $H$ comes from an identification of the fibers $f$ and $f^{\prime}$, and a sphere section $\sigma$ of self-intersection -2 obtained by sewing the sphere sections $e_{(g+1)^{2}}$ and $e_{(g+1)^{2}}^{\prime}$. The remaining $g(g-1)$ copies of $H$ come from $g(g-1)$ rim tori and their dual $(-2)$-spheres (see related discussion in [19, p. 73]). These $4 g^{2}+2 g+2$ classes generate $H_{2}$ of $W(g)$. Furthermore, using the formula for the canonical class of the generalized symplectic sum and the adjunction inequality, we compute $K_{W(g)}=(g-2)\left(h+h^{\prime}\right)$. Also, the class of the genus $g$ surface of square 2 of the genus $g$ Lefschetz pencil on $W(g)$ is given by $h+h^{\prime}$. As a consequence, the class of the genus $g$ fiber in $W(g) \# 2 \overline{\mathbb{C P}}^{2}$ is given by $h+h^{\prime}-E_{1}-E_{2}$, where $E_{1}$ and $E_{2}$ are the homology classes of the exceptional spheres of the blowups at the points $p_{1}$ and $p_{2}$, the base points of the pencil. We can also verify that the symplectic surface $\Sigma$, given by the class $h+h^{\prime}-E_{1}-E_{2}$, has genus $g$ by applying the adjunction formula to

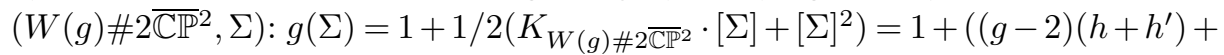
$\left.\left.E_{1}+E_{2}\right) \cdot\left(h+h^{\prime}-E_{1}-E_{2}\right)+\left(h+h^{\prime}-E_{1}-E_{2}\right)^{2}\right) / 2=1+(2(g-2)+2) / 2=g$. We can notice from the intersection form of $W(g)$ that all rim tori can be chosen to have no intersections with the genus $g$ surface in the pencil given by the homology class $h+h^{\prime}$. Thus, the genus $g$ fiber $\Sigma$ can be chosen to be disjoint from the rim tori that descend to $W(g) \# 2 \overline{\mathbb{C P}}^{2}$.

Let $k$ be any nonnegative integer, and let $\mathbb{F}_{k}$ denote the $k$ th Hirzebruch surface. Recall that $\mathbb{F}_{k}$ admits the structure of the holomorphic $\mathbb{C P}^{1}$ bundle over $\mathbb{C P}^{1}$ with two disjoint holomorphic sections $\Delta_{+k}$ and $\Delta_{-k}$ with $\Delta_{ \pm k}= \pm k$.

\section{LEMMA 13}

The genus $g$ Lefschetz fibration on $Z(g)$ over $\mathbb{S}^{2}$ with the monodromy $\left(c_{1} c_{2} \cdots\right.$ $\left.c_{2 g}\right)^{2(2 g+1)}=1$ can be obtained as the 2 -fold cover of $\mathbb{F}_{2}$ branched over the disjoint union of a smooth curve $C$ in the linear system $\left|(2 g+1) \Delta_{+2}\right|$ and $\Delta_{-2}$.

\section{Proof}

The Lefschetz fibration on $Z(g) \rightarrow \mathbb{C P}^{1}$ is obtained by composing the branchedcover map $Z(g) \rightarrow \mathbb{F}_{2}$ with the bundle map $\mathbb{F}_{2} \rightarrow \mathbb{C P}^{1}$. A generic fiber is the double cover of a sphere fiber of $\mathbb{F}_{2}$ branched over $2 g+2$ points. The monodromy of this Lefschetz fibration can be derived from the braid monodromy of the branch curve $C \cup \Delta_{-2}$. The fibration admits a holomorphic sphere section $S$ with $S^{2}=-1$, which is obtained by lifting $\Delta_{-2}$ to $Z(g)$.

\subsection{Rational blowdown}

In this section, we review the rational blowdown surgery introduced by Fintushel and Stern [14]. For details the reader is referred to [14] and [29].

Let $p \geq 2$, and let $C_{p}$ be the smooth 4-manifold obtained by plumbing disk bundles over the 2 -sphere according to the following linear diagram: 


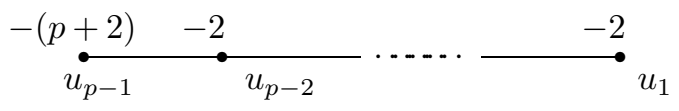

where each vertex $u_{i}$ of the linear diagram represents a disk bundle over the 2 -sphere with the given Euler number.

The boundary of $C_{p}$ is the lens space $L\left(p^{2}, 1-p\right)$, which also bounds a rational ball $B_{p}$ with $\pi_{1}\left(B_{p}\right)=\mathbb{Z}_{p}$ and $\pi_{1}\left(\partial B_{p}\right) \rightarrow \pi_{1}\left(B_{p}\right)$ surjective. If $C_{p}$ is embedded in a 4-manifold $X$, then the rational blowdown manifold $X_{p}$ is obtained by replacing $C_{p}$ with $B_{p}$, that is, $X_{p}=\left(X \backslash C_{p}\right) \cup B_{p}$. If $X$ and $X \backslash C_{p}$ are simply connected, then so is $X_{p}$. The following lemma is easy to check, so we omit the proof.

\section{LEMMA 14}

We have $b_{2}^{+}\left(X_{p}\right)=b_{2}^{+}(X), e\left(X_{p}\right)=e(X)-(p-1), \sigma\left(X_{p}\right)=\sigma(X)+(p-1)$, $c_{1}^{2}\left(X_{p}\right)=c_{1}^{2}(X)+(p-1)$, and $\chi_{h}\left(X_{p}\right)=\chi_{h}(X)$.

We now collect some theorems on rational blowdown for later use.

\section{THEOREM 15 ([14], [29])}

Suppose that $X$ is a smooth 4-manifold with $b_{2}^{+}(X)>1$ which contains a configuration $C_{p}$. If $L$ is a Seiberg-Witten invariant basic class of $X$ satisfying $L \cdot u_{i}=0$ for any $i$ with $1 \leq i \leq p-2$ and $L \cdot u_{p-1}= \pm p$, then $L$ induces an $S W$ basic class $\bar{L}$ of $X_{p}$ such that $S W_{X_{p}}(\bar{L})=S W_{X}(L)$.

\section{THEOREM 16 ([14], [29])}

If a simply connected smooth 4-manifold $X$ contains a configuration $C_{p}$, then the Seiberg-Witten invariants of $X_{p}$ are completely determined by those of $X$. That is, for any characteristic line bundle $\bar{L}$ on $X_{p}$ with $S W_{X_{p}}(\bar{L}) \neq 0$, there exists a characteristic line bundle $L$ on $X$ such that $S W_{X}(L)=S W_{X_{p}}(\bar{L})$.

THEOREM 17 ([13], [11] $(P=2)$, [12] $(P \geq 3))$

Let $\varrho, \varrho^{\prime}$ be positive relators of $\Gamma_{g}$, and let $X_{\varrho}, X_{\varrho^{\prime}}$ be the corresponding Lefschetz fibrations over $\mathbb{S}^{2}$, respectively. Suppose that $\varrho^{\prime}$ is obtained by applying a daisy substitution of type $p$ to $\varrho$. Then, $X_{\varrho^{\prime}}$ is a rational blowdown of $X_{\varrho}$ along a configuration $C_{p}$. Therefore, we have

$$
\sigma\left(X_{\varrho}^{\prime}\right)=\sigma\left(X_{\varrho}\right)+(p-1) \quad \text { and } \quad e\left(X_{\varrho}^{\prime}\right)=e\left(X_{\varrho}\right)-(p-1) .
$$

\subsection{Knot surgery}

In this section, we briefly review the knot surgery operation, which gives rise to mutually nondiffeomorphic manifolds. For details, the reader is referred to [15].

Let $X$ be a 4-manifold with $b_{2}^{+}(X)>1$ and containing a homologically essential torus $T$ of self-intersection 0 . Let $N(K)$ be a tubular neighborhood of $K$ in $\mathbb{S}^{3}$, and let $T \times D^{2}$ be a tubular neighborhood of $T$ in $X$. The knot surgery manifold 
$X_{K}$ is defined by $X_{K}=\left(X \backslash\left(T \times D^{2}\right)\right) \cup\left(\mathbb{S}^{1} \times\left(\mathbb{S}^{3} \backslash N(K)\right)\right)$ where two pieces are glued in such a way that the homology class of $\left[p t \times \partial D^{2}\right]$ is identified with $[p t \times \lambda]$, where $\lambda$ is the class of the longitude of knot $K$. Fintushel and Stern [15] proved the theorem that shows that Seiberg-Witten invariants of $X_{K}$ can be completely determined by the Seiberg-Witten invariant of $X$ and the Alexander polynomial of $K$. Moreover, if $X$ and $X \backslash T$ are simply connected, then so is $X_{K}$.

\section{THEOREM 18}

Suppose that $\pi_{1}(X)=\pi_{1}(X \backslash T)=1$, and suppose that $T$ lies in a cusp neighborhood in $X$. Then $X_{K}$ is homeomorphic to $X$ and the Seiberg-Witten invariants of $X_{K}$ are $S W_{X_{K}}=S W_{X} \cdot \Delta_{K}\left(t^{2}\right)$, where $t=t_{T}$ (in the notation of [15]) and $\Delta_{K}$ is the symmetrized Alexander polynomial of $K$. If the Alexander polynomial $\Delta_{K}(t)$ of knot $K$ is not monic, then $X_{K}$ admits no symplectic structure. Moreover, if $X$ is symplectic and $K$ is a fibered knot, then $X_{K}$ admits a symplectic structure.

\section{Lemmas}

In this section, we construct some relations by applying elementary transformations. These relations will be used to construct new relations obtained by daisy substitutions in Section 6.

Let $a_{1}, \ldots, a_{k}$ be a sequence of simple closed curves on an oriented surface such that $a_{i}$ and $a_{j}$ are disjoint if $|i-j| \geq 2$ and such that $a_{i}$ and $a_{i+1}$ intersect at one point. For simplicity of notation, we write $a_{i},{ }_{f}\left(a_{i}\right)$ instead of $t_{a_{i}}, t_{f\left(a_{i}\right)}=$ $f t_{a_{i}} f^{-1}$, respectively. Moreover, write

$$
b_{i}=a_{i+1}\left(a_{i}\right) \quad \text { and } \quad \bar{b}_{i}={ }_{a_{i+1}^{-1}}\left(a_{i}\right) .
$$

Below we denote the arrangement using the conjugation (i.e., the cyclic permutation) and the arrangement using the relation $(\mathrm{i})$ by $\stackrel{C}{\rightarrow}$ and $\stackrel{(\mathrm{i})}{\longrightarrow}$, respectively. We recall the following relations:

$$
a_{i+1} \cdot a_{i} \sim b_{i} \cdot a_{i+1} \quad \text { and } \quad a_{i} \cdot a_{i+1} \sim a_{i+1} \cdot \bar{b}_{i} .
$$

In particular, we note that

$$
a_{i} \cdot a_{j} \sim a_{j} \cdot a_{i} \text { for }|i-j|>1 .
$$

By drawing the curves, it is easy to verify that, for $m=1, \ldots, k-1$ and $i=m, \ldots, k-1$,

$$
a_{k} a_{k-1} \cdots a_{m+1} a_{m}\left(a_{i+1}\right)=a_{i} \quad \text { and } \quad a_{m} a_{m+1} \cdots a_{k-1} a_{k}\left(a_{i}\right)=a_{i+1} .
$$

Using the relation $t_{f(c)}=f t_{c} f^{-1}$, we obtain the following:

$$
\begin{aligned}
\left(a_{k} a_{k-1} \cdots a_{m+1} a_{m}\right) \cdot a_{i+1} & \sim a_{i} \cdot\left(a_{k} a_{k-1} \cdots a_{m+1} a_{m}\right), \\
\left(a_{m} a_{m+1} \cdots a_{k-1} a_{k}\right) \cdot a_{i} & \sim a_{i+1} \cdot\left(a_{m} a_{m+1} \cdots a_{k-1} a_{k}\right) .
\end{aligned}
$$




\section{LEMMA 19}

For $2 \leq k$, we have the following relations:
(a) $\left(a_{k-1} a_{k-2} \cdots a_{2} a_{1}\right) \cdot\left(a_{k} a_{k-1} \cdots a_{2} a_{1}\right) \sim a_{k}^{k} \cdot \bar{b}_{k-1} \cdots \bar{b}_{2} \bar{b}_{1}$,
(b) $\left(a_{1} a_{2} \cdots a_{k-1} a_{k}\right) \cdot\left(a_{1} a_{2} \cdots a_{k-2} a_{k-1}\right) \sim b_{1} b_{2} \cdots b_{k-1} \cdot a_{k}^{k}$.

Proof

The proof will be given by induction on $k$. Suppose that $k=2$. Then, we have

$$
a_{1} a_{2} a_{1} \stackrel{(2)}{\longrightarrow} a_{2} a_{1} a_{2} \sim a_{2}^{2} \cdot \bar{b}_{1} \text {. }
$$

Hence, the conclusion of the lemma holds for $k=2$.

Let us assume inductively that the relation holds for $k=j$. Then,

$$
\begin{aligned}
& \left(a_{j} a_{j-1} \cdots a_{1}\right) \cdot\left(a_{j+1} a_{j} \cdots a_{1}\right) \\
& \quad \sim a_{j} a_{j+1} \cdot\left(a_{j-1} \cdots a_{1}\right) \cdot\left(a_{j} \cdots a_{1}\right) \\
& \quad \sim a_{j} a_{j+1} \cdot a_{j}^{j+1} \cdot \bar{b}_{j-1} \cdots \bar{b}_{1} \\
& \quad \stackrel{(3)}{\longrightarrow} a_{j+1}^{j+1} \cdot a_{j} a_{j+1} \cdot \bar{b}_{j-1} \cdots \bar{b}_{1} \\
& \quad \sim a_{j+1}^{j+1} \cdot a_{j+1} \cdot \bar{b}_{j} \cdot \bar{b}_{j-1} \cdots \bar{b}_{1} .
\end{aligned}
$$

This proves part (a). The proof of (b) is similar and therefore omitted.

\section{LEMMA 20}

Let $l \geq 0$. We define an element $\phi$ to be

$$
\phi_{l}=a_{2 l+1}^{l+1} a_{2 l-1}^{l} \cdots a_{5}^{3} a_{3}^{2} a_{1} .
$$

Let $D$ and $E$ be two products of right-handed Dehn twists, and write them as $D=d_{1} \cdots d_{k_{1}}$ and $E=e_{1} \cdots e_{k_{2}}$, respectively. If a word $W_{1}$ is obtained by applying a sequence of the conjugation and the elementary transformations to a word $W_{2}$, then we denote it by $\sim_{C}$. For $l \geq 1$, we have the following:

(a) $D \cdot a_{2 l} \cdots a_{2} a_{1} \cdot a_{2 l+1} \cdots a_{2} a_{1} \cdot E$

$$
\sim_{C \phi_{l}}(D) \cdot\left(a_{2 l+1}^{l+1} \cdot a_{2 l} \cdots a_{2} a_{1}\right) \cdot\left(b_{2 l} \cdots b_{4} \cdot b_{2}\right) \cdot{ }_{\phi_{l}}(E),
$$

(b) $D \cdot a_{1} a_{2} \cdots a_{2 l+1} \cdot a_{1} a_{2} \cdots a_{2 l} \cdot E$

$$
\sim_{C} \phi_{l}^{-1}(D) \cdot\left(\bar{b}_{2} \cdot \bar{b}_{4} \cdots \bar{b}_{2 l}\right) \cdot\left(a_{1} a_{2} \cdots a_{2 l} \cdot a_{2 l+1}^{l+1}\right) \cdot{ }_{\phi_{l}^{-1}}(E),
$$

where ${ }_{\phi_{l}}(D)={ }_{\phi_{l}}\left(d_{1}\right) \cdots_{\phi_{l}}\left(d_{k_{1}}\right)$ and ${ }_{\phi_{l}}(E)={ }_{\phi_{l}}\left(e_{1}\right) \cdots_{\phi_{l}}\left(e_{k_{2}}\right)$.

Proof

For $1 \leq m \leq 2 l-1$ and $m \leq i \leq 2 l-1$, we have the following equalities from (2) and (3): 


$$
\begin{aligned}
& \left(a_{2 l} \cdots a_{1} \cdot a_{2 l+1} \cdots a_{m}\right) \cdot a_{i+2} \sim a_{i} \cdot\left(a_{2 l} \cdots a_{1} \cdot a_{2 l+1} \cdots a_{m}\right), \\
& a_{i+2} \cdot\left(a_{m} \cdots a_{2 l+1} \cdot a_{1} \cdots a_{2 l}\right) \sim\left(a_{m} \cdots a_{2 l+1} \cdot a_{1} \cdots a_{2 l}\right) \cdot a_{i} .
\end{aligned}
$$

We first show (a). Since

$$
\begin{aligned}
& \phi_{l-1}(D) \cdot\left(a_{2 l} a_{2 l-1} \cdots a_{1}\right) \cdot\left(b_{2 l} b_{2 l-2} \cdots b_{2}\right) \cdot a_{2 l+1}^{l+1} \cdot \phi_{l-1}(E) \\
& \quad{ }^{C}{ }_{a_{2 l+1}^{l+1} \phi_{l-1}}(D) \cdot a_{2 l+1}^{l+1} \cdot\left(a_{2 l} a_{2 l-1} \cdots a_{1}\right) \cdot\left(b_{2 l} b_{2 l-2} \cdots b_{2}\right) \cdot a_{a_{2 l+1}^{l+1} \phi_{l-1}}(E) \\
& \quad={ }_{\phi_{l}}(D) \cdot a_{2 l+1}^{l+1} \cdot\left(a_{2 l} a_{2 l-1} \cdots a_{1}\right) \cdot\left(b_{2 l} b_{2 l-2} \cdots b_{2}\right) \cdot{ }_{\phi_{l}}(E),
\end{aligned}
$$

it is sufficient to prove

$$
\begin{aligned}
D & \cdot\left(a_{2 l} a_{2 l-1} \cdots a_{1}\right) \cdot\left(a_{2 l+1} a_{2 l} \cdots a_{1}\right) \cdot E \\
& \sim{ }_{\phi_{l-1}}(D) \cdot\left(a_{2 l} a_{2 l-1} \cdots a_{1}\right) \cdot\left(b_{2 l} b_{2 l-2} \cdots b_{2}\right) \cdot a_{2 l+1}^{l+1} \cdot \phi_{l-1}(E) .
\end{aligned}
$$

The proof is by induction on $l$. Suppose that $l=0$. Then, we have

$$
\begin{aligned}
D & \cdot a_{2} a_{1} \cdot a_{3} a_{2} a_{1} \cdot E \\
& \quad{ }^{C} a_{1}(D) \cdot a_{1} \cdot a_{2} a_{1} \cdot a_{3} a_{2} \cdot a_{1}(E) \\
& \stackrel{(4)}{\longrightarrow} a_{1}(D) \cdot a_{2} a_{1} \cdot a_{3} a_{2} \cdot a_{3} \cdot a_{1}(E) \\
& \sim a_{1}(D) \cdot a_{2} a_{1} \cdot b_{2} \cdot a_{3} \cdot a_{3} \cdot a_{1}(E) .
\end{aligned}
$$

Since $\phi_{0}=a_{1}$, the conclusion of the lemma holds for $l=0$.

Let us assume, inductively, that the relation holds for $l=j$. Note that since $\phi_{j-1}\left(a_{2 j+2}\right)=a_{2 j+2}$ and $\phi_{j-1}\left(a_{2 j+3}\right)=a_{2 j+3}$, we have

$$
{ }_{\phi_{j-1}}\left(D a_{2 j+2} a_{2 j+1} a_{2 j+3} a_{2 j+2}\right)={ }_{\phi_{j-1}}(D) a_{2 j+2} a_{2 j+1} a_{2 j+3} a_{2 j+2} .
$$

Since $a_{2 j+3}$ is disjoint from $b_{2}, b_{4}, \ldots, b_{2 j}$ and $\phi_{j}=a_{2 j+1}^{j+1} \phi_{j-1}$, we have

$$
\begin{aligned}
D & \cdot\left(a_{2 j+2} a_{2 j+1} a_{2 j} \cdots a_{1}\right) \cdot\left(a_{2 j+3} a_{2 j+2} a_{2 j+1} \cdots a_{1}\right) \cdot E \\
& \sim D \cdot a_{2 j+2} a_{2 j+1} a_{2 j+3} a_{2 j+2} \cdot\left(a_{2 j} \cdots a_{1}\right) \cdot\left(a_{2 j+1} \cdots a_{1}\right) \cdot E \\
& \sim{ }_{\phi_{j-1}}(D) \cdot a_{2 j+2} a_{2 j+1} a_{2 j+3} a_{2 j+2} \cdot\left(a_{2 j} \cdots a_{1}\right) \cdot\left(b_{2 j} \cdots b_{2}\right) \cdot a_{2 j+1}^{j+1} \cdot \phi_{j-1}(E) \\
& { }_{C} \phi_{\phi_{j}}(D) \cdot a_{2 j+1}^{j+1} \cdot a_{2 j+2} a_{2 j+1} a_{2 j+3} a_{2 j+2} \cdot\left(a_{2 j} \cdots a_{1}\right) \cdot\left(b_{2 j} \cdots b_{2}\right) \cdot \phi_{j}(E) \\
& \sim{ }_{\phi_{j}}(D) \cdot a_{2 j+1}^{j+1} \cdot\left(a_{2 j+2} a_{2 j+1} a_{2 j} \cdots a_{1}\right) \cdot a_{2 j+3} a_{2 j+2} \cdot\left(b_{2 j} \cdots b_{2}\right) \cdot{ }_{\phi_{j}}(E) \\
& \stackrel{(4)}{\longrightarrow} \phi_{j}(D) \cdot\left(a_{2 j+2} a_{2 j+1} a_{2 j} \cdots a_{1}\right) \cdot a_{2 j+3} a_{2 j+2} \cdot a_{2 j+3}^{j+1} \cdot\left(b_{2 j} \cdots b_{2}\right) \cdot \phi_{j}(E) \\
& \sim{ }_{\phi_{j}}(D) \cdot\left(a_{2 j+2} a_{2 j+1} a_{2 j} \cdots a_{1}\right) \cdot b_{2 j+2} \cdot a_{2 j+3} \cdot a_{2 j+3}^{j+1} \cdot\left(b_{2 j} \cdots b_{2}\right) \cdot \phi_{\phi_{j}}(E) \\
& \sim{ }_{\phi_{j}}(D) \cdot\left(a_{2 j+2} a_{2 j+1} a_{2 j} \cdots a_{1}\right) \cdot\left(b_{2 j+2} b_{2 j} \cdots b_{2}\right) \cdot a_{2 j+3}^{j+2} \cdot \phi_{j}(E) .
\end{aligned}
$$

This proves part (a) of the lemma. The proof of part (b) is similar and left to the reader. 


\section{A lift of hyperelliptic relations}

In this section, we construct a relation which gives a lift of a relation (and which is Hurwitz equivalent to $I(g)$ ) from $\Gamma_{g}$ to $\Gamma_{g}^{1}$. This relation will be used in Section 6 .

Suppose that $g \geq 2$. Let $\Sigma_{g}^{n}$ be the surface of genus $g$ with $b$ boundary components $\delta_{1}, \delta_{2}, \ldots, \delta_{n}$. Let $\alpha_{1}, \alpha_{2}, \ldots, \alpha_{2 g}, \alpha_{2 g+1}, \alpha_{2 g+1}^{\prime}$ and $\delta, \zeta_{1}, \ldots, \zeta_{n}$ be the simple closed curves as shown in Figure 4.

\section{LEMMA 21}

The following relation holds in $\Gamma_{g}^{n}$ :

$$
\left(\alpha_{1} \alpha_{2} \cdots \alpha_{2 g}\right)^{2 g+1}=\left(\alpha_{1} \alpha_{2} \cdots \alpha_{2 g-1}\right)^{2 g} \cdot \alpha_{2 g} \cdots \alpha_{2} \alpha_{1} \alpha_{1} \alpha_{2} \cdots \alpha_{2 g} .
$$

\section{Proof}

The proof follows from the braid relations, $\alpha_{i} \cdot \alpha_{i+1} \cdot \alpha_{i}=\alpha_{i+1} \cdot \alpha_{i} \cdot \alpha_{i+1}$ and $\alpha_{i} \cdot \alpha_{j}=\alpha_{j} \cdot \alpha_{i}$ for $|i-j|>1$.

\section{LEMMA 22}

The following relation holds in $\Gamma_{g}^{n}$ :

$$
\delta=\left(\alpha_{2 g+1} \alpha_{2 g} \cdots \alpha_{2} \alpha_{1} \alpha_{1} \alpha_{2} \cdots \alpha_{2 g} \alpha_{2 g+1}^{\prime}\right)^{2} .
$$

In particular, since $\delta_{1}=\delta$ for $n=1$, this relation is a lift of $H(g)$ from $\Gamma_{g}$ to $\Gamma_{g}^{1}$.

\section{Proof}

A regular neighborhood of $\alpha_{1} \cup \alpha_{2} \cup \cdots \cup a_{2 g-1}$ is a subsurface of genus $g-1$ with two boundary components, $\alpha_{2 g+1}$ and $\alpha_{2 g+1}^{\prime}$. Moreover, a regular neighborhood of $\alpha_{1} \cup \alpha_{2} \cup \cdots \cup a_{2 g}$ is a subsurface of genus $g$ with one boundary component $\delta$. Then, it is well known that the following relations, called the chain relations, hold:

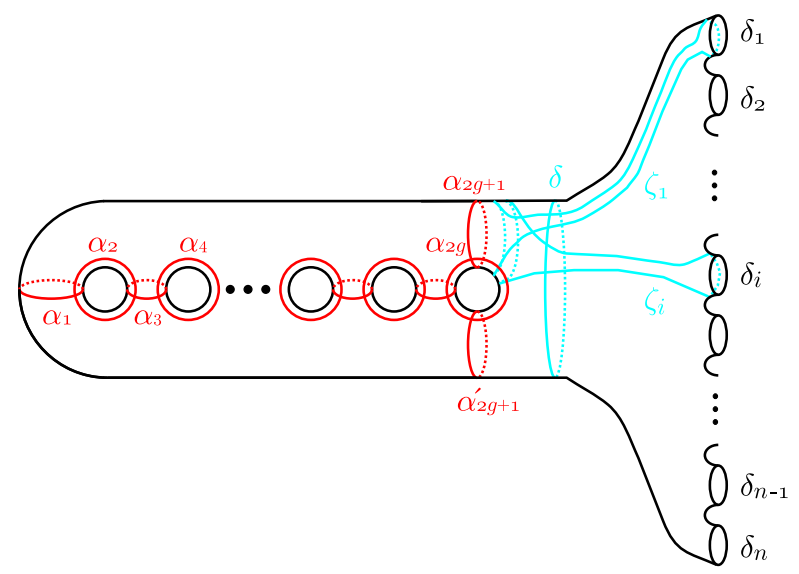

Figure 4. The curves $\alpha_{1}, \ldots, \alpha_{2 g+1}, \alpha_{2 g+1}^{\prime}, \delta, \zeta_{1}, \ldots, \zeta_{n}$ on $\Sigma_{g}^{n}$ and the boundary components $\delta_{1}, \ldots, \delta_{n}$ of $\Sigma_{g}^{n}$. 


$$
\alpha_{2 g+1} \alpha_{2 g+1}^{\prime}=\left(\alpha_{1} \cdots \alpha_{2 g-1}\right)^{2 g}, \quad \delta=\left(\alpha_{1} \alpha_{2} \alpha_{3} \cdots \alpha_{2 g}\right)^{4 g+2} .
$$

By applying the relations above and Lemma 21, we obtain the following relation:

$$
\delta=\left(\alpha_{2 g+1} \alpha_{2 g+1}^{\prime} \alpha_{2 g} \cdots \alpha_{2} \alpha_{1} \alpha_{1} \alpha_{2} \cdots \alpha_{2 g}\right)^{2} .
$$

Since $\alpha_{2 g+1}^{\prime}$ is disjoint from $\alpha_{2 g+1}$, by the conjugation of $\alpha_{2 g+1}^{\prime}$, we obtain the claim. If $n=1$, then it is easily seen that this is a lift of $H(g)$ from $\Gamma_{g}$ to $\Gamma_{g}^{1}$. This completes the proof.

\section{LEMMA 23}

The following relation holds in $\Gamma_{g}^{n}$ :

$$
\begin{aligned}
& \left(\alpha_{2 g+1} \alpha_{2 g} \cdots \alpha_{2} \alpha_{1} \alpha_{1} \alpha_{2} \cdots \alpha_{2 g} \alpha_{2 g+1}^{\prime}\right)^{2} \\
& \quad \sim\left(\alpha_{2 g+1} \alpha_{2 g} \cdots \alpha_{2} \alpha_{1}\right)^{2} \cdot\left(\alpha_{1} \alpha_{2} \cdots \alpha_{2 g} \alpha_{2 g+1}\right) \cdot\left(\alpha_{1} \alpha_{2} \cdots \alpha_{2 g} \alpha_{2 g+1}^{\prime}\right) .
\end{aligned}
$$

Proof

By drawing a picture, we find that, for each $i=1,2, \ldots, 2 g$,

$$
\begin{aligned}
\alpha_{2 g+1} \cdots \alpha_{2} \alpha_{1} \alpha_{1} \alpha_{2} \cdots \alpha_{2 g+1}\left(\alpha_{i}\right) & =\alpha_{i}, \\
\alpha_{2 g} \cdots \alpha_{2} \alpha_{1} \alpha_{1} \alpha_{2} \cdots \alpha_{2 g}\left(\alpha_{2 g+1}^{\prime}\right) & =\alpha_{2 g+1} .
\end{aligned}
$$

These give the following relations:

$$
\begin{gathered}
\alpha_{2 g+1} \cdots \alpha_{2} \alpha_{1} \alpha_{1} \alpha_{2} \cdots \alpha_{2 g+1} \cdot \alpha_{i} \sim \alpha_{i} \cdot \alpha_{2 g+1} \cdots \alpha_{2} \alpha_{1} \alpha_{1} \alpha_{2} \cdots \alpha_{2 g+1}, \\
\alpha_{2 g} \cdots \alpha_{2} \alpha_{1} \alpha_{1} \alpha_{2} \cdots \alpha_{2 g} \cdot \alpha_{2 g+1}^{\prime} \sim \alpha_{2 g+1} \cdot \alpha_{2 g} \cdots \alpha_{2} \alpha_{1} \alpha_{1} \alpha_{2} \cdots \alpha_{2 g} .
\end{gathered}
$$

From these relations, we have

$$
\begin{aligned}
& \alpha_{2 g+1} \alpha_{2 g} \cdots \alpha_{2} \alpha_{1} \alpha_{1} \alpha_{2} \cdots \alpha_{2 g} \alpha_{2 g+1}^{\prime} \alpha_{2 g+1} \alpha_{2 g} \cdots \alpha_{2} \alpha_{1} \alpha_{1} \alpha_{2} \cdots \alpha_{2 g} \alpha_{2 g+1}^{\prime} \\
& \stackrel{(7)}{\longrightarrow} \alpha_{2 g+1} \cdot \alpha_{2 g+1} \alpha_{2 g} \cdots \alpha_{2} \alpha_{1} \alpha_{1} \alpha_{2} \cdots \alpha_{2 g} \alpha_{2 g+1} \cdot \alpha_{2 g} \cdots \alpha_{2} \alpha_{1} \\
& \quad \cdot \alpha_{1} \alpha_{2} \cdots \alpha_{2 g} \alpha_{2 g+1}^{\prime} \\
& \stackrel{(6)}{\longrightarrow}\left(\alpha_{2 g+1} \alpha_{2 g} \cdots \alpha_{2} \alpha_{1}\right) \cdot\left(\alpha_{2 g+1} \alpha_{2 g} \cdots \alpha_{2} \alpha_{1} \alpha_{1} \alpha_{2} \cdots \alpha_{2 g} \alpha_{2 g+1}\right) \\
& \cdot\left(\alpha_{1} \alpha_{2} \cdots \alpha_{2 g} \alpha_{2 g+1}^{\prime}\right) .
\end{aligned}
$$

This completes the proof.

\section{New words in the mapping class group via daisy relation}

We define $\phi$ in $\Gamma_{g}^{n}$ to be

$$
\phi=\alpha_{2 g+1}^{g+1} \alpha_{2 g-1}^{g} \cdots \alpha_{5}^{3} \alpha_{3}^{2} \alpha_{1} .
$$

Note that $\phi\left(\alpha_{2 i-1}\right)=\alpha_{2 i-1}$ for each $i=1, \ldots, g+1$ and $\phi\left(\alpha_{2 g+1}^{\prime}\right)=\alpha_{2 g+1}^{\prime}$. For simplicity of notation, we write

$$
\beta_{i}=\alpha_{i+1}\left(\alpha_{i}\right), \quad \bar{\beta}_{i}={ }_{\alpha_{i+1}^{-1}}\left(\alpha_{i}\right), \quad \gamma_{i+1}={ }_{\alpha_{i}}\left(\alpha_{i+1}\right), \quad \bar{\gamma}_{i+1}={ }_{\alpha_{i}^{-1}}\left(\alpha_{i+1}\right) .
$$




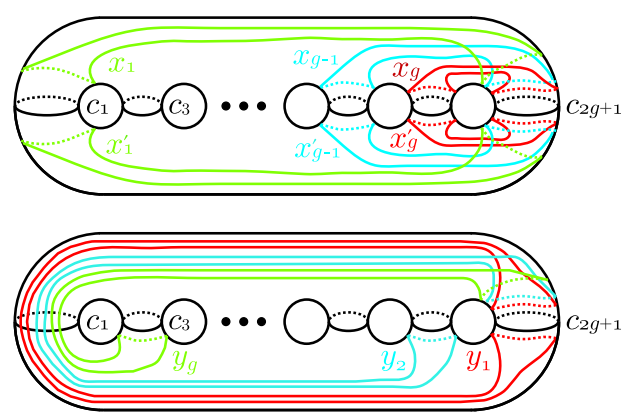

Figure 5. The curves $x_{1}, \ldots, x_{g}, x_{1}^{\prime}, \ldots, x_{g}^{\prime}$, and $y_{1}, \ldots, y_{g}$ on $\Sigma_{g}$.

We denote by $\varphi, d_{i}, \bar{d}_{i}, e_{i+1}$, and $\bar{e}_{i+1}$ the images of $\phi, \beta_{i}, \bar{\beta}_{i}, \gamma_{i+1}$ and $\bar{\gamma}_{i+1}$ under the map $\Gamma_{g}^{n} \rightarrow \Gamma_{g}$; that is,

$$
\begin{aligned}
\varphi & =c_{2 g+1}^{g+1} c_{2 g-1}^{g} \cdots c_{5}^{3} c_{3}^{2} c_{1}, \\
d_{i} & ={ }_{c_{i+1}}\left(c_{i}\right), \quad \bar{d}_{i}={ }_{c_{i+1}^{-1}}\left(c_{i}\right), \quad e_{i+1}={ }_{c_{i}}\left(c_{i+1}\right), \quad \text { and } \quad \bar{e}_{i+1}={ }_{c_{i}^{-1}}\left(c_{i+1}\right) .
\end{aligned}
$$

Then, note that $\varphi\left(c_{2 i-1}\right)=c_{2 i-1}$ for each $i=1, \ldots, g+1$. If a word $W_{1}$ is obtained by applying simultaneous conjugations by $\psi$ to a word $W_{2}$, then we denote it by $\stackrel{\psi}{\rightarrow}$.

Let $x_{1}, \ldots, x_{g}, x_{1}^{\prime}, \ldots, x_{g}^{\prime}$, and $y_{1}, \ldots, y_{g}$ be the simple closed curves on $\Sigma_{g}$ given in Figure 5 . Moreover, we define $y_{g+1}, \ldots, y_{2 g-1}$ to be $x_{2}, \ldots, x_{g}$, respectively. We take the following two daisy relators of type $g-1$ in $\Gamma_{g}$ :

$$
\begin{aligned}
& D_{g-1}:=c_{1}^{-1} c_{3}^{-1} \cdots c_{2 g-1}^{-1} \cdot c_{2 g+1}^{-(g-2)} \cdot x_{1} x_{2} \cdots x_{g}, \\
& D_{g-1}^{\prime}:=c_{1}^{-1} c_{3}^{-1} \cdots c_{2 g-1}^{-1} \cdot c_{2 g+1}^{-(g-2)} \cdot x_{1}^{\prime} x_{2}^{\prime} \cdots x_{g}^{\prime},
\end{aligned}
$$

and the following daisy relator of type $2(g-1)$ :

$$
\begin{aligned}
D_{2(g-1)} & :=c_{2 g+1}^{-1} c_{2 g-1}^{-1} \cdots c_{5}^{-1} c_{3}^{-1} \cdot c_{3}^{-1} c_{5}^{-1} \cdots c_{2 g-1}^{-1} \cdot c_{2 g+1}^{-2 g+3} \cdot y_{1} y_{2} \cdots y_{2 g-1} \\
& =c_{3}^{-2} c_{5}^{-2} \cdots c_{2 g-1}^{-2} \cdot c_{2 g+1}^{-2 g+2} \cdot y_{1} y_{2} \cdots y_{2 g-1} .
\end{aligned}
$$

Let $\chi_{1}, \ldots, \chi_{g}$ be the simple closed curves on $\Sigma_{g}^{n}$ given in Figure 6 . We denote by $\mathcal{D}_{g-1}$ the following daisy relator of type $g-1$ in $\Gamma_{g}^{n}$ :

$$
\mathcal{D}_{g-1}=\alpha_{1}^{-1} \alpha_{3}^{-1} \cdots \alpha_{2 g-1}^{-1} \cdot \alpha_{2 g+1}^{-(g-2)} \cdot \chi_{1} \chi_{2} \cdots \chi_{g} .
$$

Since it is easily seen that $\alpha_{i}$ and $\chi_{i}$ are mapped to $c_{i}$ and $x_{i}$ under the map $\Gamma_{g}^{n} \rightarrow \Gamma_{g}$, we see that the image of this map of $\mathcal{D}_{g-1}$ is $D_{g-1}$.

\section{THEOREM 24}

There is a positive relator

$$
H(g, 1)={ }_{\varphi^{-1}}\left(\bar{d}_{2 g}\right) \cdots \varphi_{\varphi^{-1}}\left(\bar{d}_{2}\right)_{\varphi^{-1}}\left(\bar{d}_{1}\right) \bar{d}_{2} \bar{d}_{4} \cdots \bar{d}_{2 g} e_{2} e_{4} \cdots e_{2 g} x_{1} x_{2} \cdots x_{g} c_{2 g+1}^{2 g+6},
$$

which is obtained by applying $D_{g-1}$-substitution once to $H(g)$. Moreover, the Lefschetz fibration $f_{H(g, 1)}$ has $2 g+6$ disjoint sections of self-intersection -1 . 


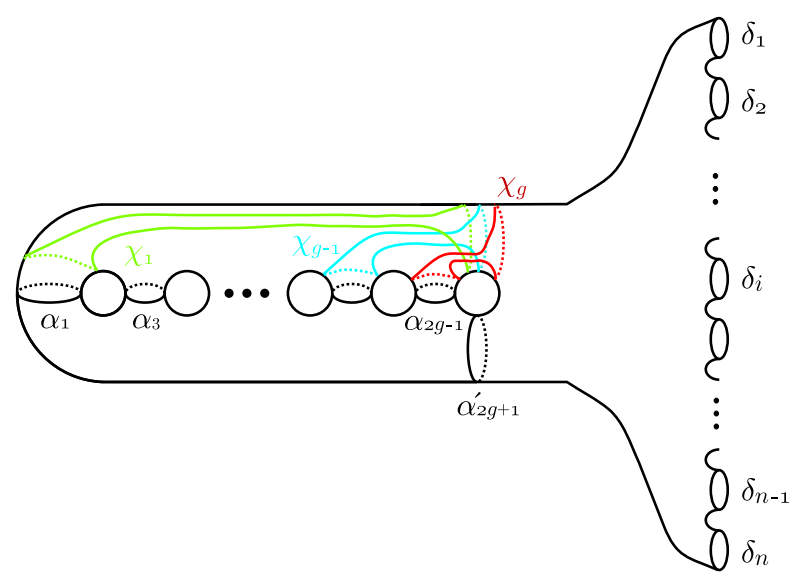

Figure 6. The curves $\chi_{1}, \ldots, \chi_{g}$ on $\Sigma_{g}^{n}$.

Proof

Note that by Lemma 19(a), we have

$$
\begin{aligned}
\left(\alpha_{2 g+1} \alpha_{2 g} \cdots \alpha_{1}\right)^{2} & =\alpha_{2 g+1} \cdot\left(\alpha_{2 g} \cdots \alpha_{1}\right) \cdot\left(\alpha_{2 g+1} \alpha_{2 g} \cdots \alpha_{1}\right) \\
& \sim \alpha_{2 g+1}^{2 g+2} \bar{\beta}_{2 g} \cdots \bar{\beta}_{1} .
\end{aligned}
$$

Moreover, one can check that

$$
\alpha_{1} \alpha_{2} \alpha_{3} \cdots \alpha_{2 g} \sim\left(\gamma_{2} \gamma_{4} \gamma_{6} \cdots \gamma_{2 g}\right) \cdot\left(\alpha_{1} \alpha_{3} \alpha_{5} \cdots \alpha_{2 g-1}\right) .
$$

Therefore, by Lemma 20(b), we have

$$
\begin{aligned}
& \left(\alpha_{2 g+1} \alpha_{2 g} \cdots \alpha_{2} \alpha_{1}\right)^{2} \cdot\left(\alpha_{1} \alpha_{2} \cdots \alpha_{2 g} \alpha_{2 g+1}\right) \cdot\left(\alpha_{1} \alpha_{2} \cdots \alpha_{2 g} \alpha_{2 g+1}^{\prime}\right) \\
& \quad \stackrel{(8)}{\longrightarrow} \alpha_{2 g+1}^{2 g+2} \bar{\beta}_{2 g} \cdots \bar{\beta}_{1} \cdot\left(\alpha_{1} \alpha_{2} \cdots \alpha_{2 g} \alpha_{2 g+1}\right) \cdot\left(\alpha_{1} \alpha_{2} \cdots \alpha_{2 g}\right) \alpha_{2 g+1}^{\prime} \\
& \sim_{C \phi^{-1}}\left\{\alpha_{2 g+1}^{2 g+2} \cdot \bar{\beta}_{2 g} \cdots \bar{\beta}_{1}\right\} \cdot\left(\bar{\beta}_{2} \bar{\beta}_{4} \cdots \bar{\beta}_{2 g}\right) \cdot\left(\alpha_{1} \alpha_{2} \cdots \alpha_{2 g} \cdot \alpha_{2 g+1}^{g+1}\right) \cdot \alpha_{2 g+1}^{\prime} \\
& =\alpha_{2 g+1}^{2 g+2} \cdot{ }_{\phi^{-1}}\left(\bar{\beta}_{2 g}\right) \cdots \phi_{\phi^{-1}}\left(\bar{\beta}_{1}\right) \cdot\left(\bar{\beta}_{2} \bar{\beta}_{4} \cdots \bar{\beta}_{2 g}\right) \cdot\left(\alpha_{1} \alpha_{2} \cdots \alpha_{2 g} \cdot \alpha_{2 g+1}^{g+1}\right) \cdot \alpha_{2 g+1}^{\prime} \\
& \quad \stackrel{C}{\longrightarrow}_{\phi^{-1}}\left(\bar{\beta}_{2 g}\right) \cdots \phi_{\phi^{-1}}\left(\bar{\beta}_{1}\right) \cdot\left(\bar{\beta}_{2} \bar{\beta}_{4} \cdots \bar{\beta}_{2 g}\right) \cdot\left(\alpha_{1} \alpha_{2} \cdots \alpha_{2 g}\right) \cdot \alpha_{2 g+1}^{3 g+3} \cdot \alpha_{2 g+1}^{\prime} \\
& \stackrel{(9)}{\longrightarrow} \phi^{-1}\left(\bar{\beta}_{2 g}\right) \cdots \phi_{\phi^{-1}}\left(\bar{\beta}_{1}\right) \cdot\left(\bar{\beta}_{2} \bar{\beta}_{4} \cdots \bar{\beta}_{2 g}\right) \cdot\left(\gamma_{2} \gamma_{4} \cdots \gamma_{2 g}\right) \cdot\left(\alpha_{1} \alpha_{3} \cdots \alpha_{2 g-1}\right) \\
& \quad \alpha_{2 g+1}^{3 g+3} \cdot \alpha_{2 g+1}^{\prime} .
\end{aligned}
$$

Since $\delta$ is a central element of the group generated by $\alpha_{1}, \ldots, \alpha_{2 g+1}, \alpha_{2 g+1}^{\prime}$, by Lemma 23, the operation $\sim_{C}$ is Hurwitz equivalent (e.g., see [5, Lemma 6]). We have the following relation in $\Gamma_{g}^{2 g+6}$ which is Hurwitz equivalent to the relation $\delta=\left(\alpha_{2 g+1} \alpha_{2 g} \cdots \alpha_{2} \alpha_{1} \alpha_{1} \alpha_{2} \cdots \alpha_{2 g} \alpha_{2 g+1}^{\prime}\right)^{2}$ :

$$
\begin{aligned}
\delta= & { }_{\phi^{-1}}\left(\bar{\beta}_{2 g}\right) \cdots{ }_{\phi^{-1}}\left(\bar{\beta}_{1}\right) \cdot\left(\bar{\beta}_{2} \bar{\beta}_{4} \cdots \bar{\beta}_{2 g}\right) \cdot\left(\gamma_{2} \gamma_{4} \cdots \gamma_{2 g}\right) \cdot\left(\alpha_{1} \alpha_{3} \cdots \alpha_{2 g-1}\right) \\
& \cdot \alpha_{2 g+1}^{3 g+3} \cdot \alpha_{2 g+1}^{\prime} .
\end{aligned}
$$


By applying $\mathcal{D}_{g-1}$-substitution once to this relation, we have the following relation:

$$
\begin{aligned}
\delta={ }_{\phi^{-1}}\left(\bar{\beta}_{2 g}\right) \cdots_{\phi^{-1}}\left(\bar{\beta}_{1}\right) \cdot\left(\bar{\beta}_{2} \bar{\beta}_{4} \cdots \bar{\beta}_{2 g}\right) \cdot\left(\gamma_{2} \gamma_{4} \cdots \gamma_{2 g}\right) \cdot\left(\chi_{1} \chi_{2} \cdots \chi_{g}\right) \\
\cdot \alpha_{2 g+1}^{2 g+5} \cdot \alpha_{2 g+1}^{\prime} .
\end{aligned}
$$

Moreover, by $\alpha_{2 g+1}^{2 g+5} \cdot \alpha_{2 g+1}^{\prime} \cdot \delta_{1} \delta_{2} \cdots \delta_{2 g+6}=\alpha_{2 g+1}^{2 g+5} \cdot \delta_{1} \delta_{2} \cdots \delta_{2 g+6} \cdot \alpha_{2 g+1}^{\prime}$ and the daisy relation $\alpha_{2 g+1}^{2 g+5} \cdot \delta_{1} \delta_{2} \cdots \delta_{2 g+6} \cdot \alpha_{2 g+1}^{\prime}=\zeta_{1} \zeta_{2} \cdots \zeta_{2 g+6} \cdot \delta$, we obtain

$$
\begin{aligned}
\delta \cdot & \delta_{1} \delta_{2} \cdots \delta_{2 g+6} \\
= & { }_{\phi^{-1}}\left(\bar{\beta}_{2 g}\right) \cdots{ }_{\phi^{-1}}\left(\bar{\beta}_{1}\right) \cdot\left(\bar{\beta}_{2} \bar{\beta}_{4} \cdots \bar{\beta}_{2 g}\right) \cdot\left(\gamma_{2} \gamma_{4} \cdots \gamma_{2 g}\right) \cdot\left(\chi_{1} \chi_{2} \cdots \chi_{g}\right) \\
& \cdot \alpha_{2 g+1}^{2 g+5} \cdot \alpha_{2 g+1}^{\prime} \cdot \delta_{1} \delta_{2} \cdots \delta_{2 g+6} \\
= & { }_{\phi^{-1}}\left(\bar{\beta}_{2 g}\right) \cdots{ }_{\phi^{-1}}\left(\bar{\beta}_{1}\right) \cdot\left(\bar{\beta}_{2} \bar{\beta}_{4} \cdots \bar{\beta}_{2 g}\right) \cdot\left(\gamma_{2} \gamma_{4} \cdots \gamma_{2 g}\right) \cdot\left(\chi_{1} \chi_{2} \cdots \chi_{g}\right) \\
& \cdot \zeta_{1} \zeta_{2} \cdots \zeta_{2 g+6} \cdot \delta .
\end{aligned}
$$

Therefore, we have the following relation in $\Gamma_{g}^{2 g+6}$ :

$$
\begin{aligned}
\delta_{1} & \cdot \delta_{2} \cdots \delta_{2 g+6} \\
= & { }_{\phi^{-1}}\left(\bar{\beta}_{2 g}\right) \cdots{ }_{\phi^{-1}}\left(\bar{\beta}_{1}\right) \cdot\left(\bar{\beta}_{2} \bar{\beta}_{4} \cdots \bar{\beta}_{2 g}\right) \cdot\left(\gamma_{2} \gamma_{4} \cdots \gamma_{2 g}\right) \cdot\left(\chi_{1} \chi_{2} \cdots \chi_{g}\right) \\
& \cdot \zeta_{1} \zeta_{2} \cdots \zeta_{2 g+6} .
\end{aligned}
$$

It is easily seen that $\zeta_{1}, \ldots, \zeta_{2 g+6}$ are mapped to $c_{2 g+1}$ under the map $\Gamma_{g}^{2 g+6} \rightarrow \Gamma_{g}$. This completes the proof.

\section{THEOREM 25}

There is a positive relator

$$
\begin{aligned}
H(g, 2)= & { }^{-2}\left(\bar{e}_{2 g}\right) \cdots \varphi_{\varphi^{-2}}\left(\bar{e}_{4}\right)_{\varphi^{-2}}\left(\bar{e}_{2}\right)_{\varphi^{-2}}\left(d_{2 g}\right) \cdots_{\varphi^{-2}}\left(d_{4}\right) \cdot{ }_{\varphi^{-2}}\left(d_{2}\right) \\
& \cdot \bar{d}_{2} \bar{d}_{4} \cdots \bar{d}_{2 g} e_{2} e_{4} \cdots e_{2 g}\left(x_{1} x_{2} \cdots x_{g}\right)^{2} c_{2 g+1}^{8}
\end{aligned}
$$

which is obtained by applying $D_{g-1}$-substitutions twice to $H(g)$. Moreover, the Lefschetz fibration $f_{H(g, 2)}$ has eight disjoint sections of self-intersection -1 .

\section{Proof}

Let $D$ be a product of Dehn twists. Then, we note that by Lemma 20(a) we have

$$
\begin{aligned}
\left(\alpha_{2 g+1} \alpha_{2 g} \cdots \alpha_{2} \alpha_{1}\right)^{2} \cdot D & =\alpha_{2 g+1} \cdot\left(\alpha_{2 g} \cdots \alpha_{1}\right) \cdot\left(\alpha_{2 g+1} \alpha_{2 g} \cdots \alpha_{1}\right) \cdot D \\
& \sim_{C} \alpha_{2 g+1}^{g+2} \cdot\left(\alpha_{2 g} \cdots \alpha_{2} \alpha_{1}\right) \cdot\left(\beta_{2 g} \cdots \beta_{4} \beta_{2}\right) \cdot{ }_{\phi}(D) .
\end{aligned}
$$

Moreover, we have

$$
\alpha_{2 g} \cdots \alpha_{3} \alpha_{2} \alpha_{1} \sim\left(\alpha_{2 g-1} \cdots \alpha_{5} \alpha_{3} \alpha_{1}\right) \cdot\left(\bar{\gamma}_{2 g} \cdots \bar{\gamma}_{6} \bar{\gamma}_{4} \bar{\gamma}_{2}\right) .
$$

Then, by Lemma 20(b) we have

$$
\begin{aligned}
& \left(\alpha_{2 g+1} \alpha_{2 g} \cdots \alpha_{2} \alpha_{1}\right)^{2} \cdot\left(\alpha_{1} \alpha_{2} \cdots \alpha_{2 g} \alpha_{2 g+1}\right) \cdot\left(\alpha_{1} \alpha_{2} \cdots \alpha_{2 g} \alpha_{2 g+1}^{\prime}\right) \\
& \quad \stackrel{(10)}{\longrightarrow} \alpha_{2 g+1}^{g+2} \cdot\left(\alpha_{2 g} \cdots \alpha_{2} \alpha_{1}\right) \cdot\left(\beta_{2 g} \cdots \beta_{4} \beta_{2}\right)
\end{aligned}
$$




$$
\begin{aligned}
& \cdot{ }_{\phi}\left\{\left(\alpha_{1} \alpha_{2} \cdots \alpha_{2 g} \alpha_{2 g+1}\right) \cdot\left(\alpha_{1} \alpha_{2} \cdots \alpha_{2 g} \alpha_{2 g+1}^{\prime}\right)\right\} \\
& \stackrel{(11)}{\longrightarrow} \alpha_{2 g+1}^{g+2} \cdot\left(\alpha_{2 g-1} \cdots \alpha_{3} \alpha_{1}\right) \cdot\left(\bar{\gamma}_{2 g} \cdots \bar{\gamma}_{4} \bar{\gamma}_{2}\right) \cdot\left(\beta_{2 g} \cdots \beta_{4} \beta_{2}\right) \\
& \cdot{ }_{\phi}\left\{\left(\alpha_{1} \alpha_{2} \cdots \alpha_{2 g} \alpha_{2 g+1}\right) \cdot\left(\alpha_{1} \alpha_{2} \cdots \alpha_{2 g} \alpha_{2 g+1}^{\prime}\right)\right\} \\
& {\stackrel{\phi^{-1}}{\longrightarrow}}_{\phi^{-1}}\left\{\alpha_{2 g+1}^{g+2} \cdot\left(\alpha_{2 g-1} \cdots \alpha_{3} \alpha_{1}\right) \cdot\left(\bar{\gamma}_{2 g} \cdots \bar{\gamma}_{4} \bar{\gamma}_{2}\right) \cdot\left(\beta_{2 g} \cdots \beta_{4} \beta_{2}\right)\right\} \\
& \cdot\left(\alpha_{1} \alpha_{2} \cdots \alpha_{2 g} \alpha_{2 g+1}\right) \cdot\left(\alpha_{1} \alpha_{2} \cdots \alpha_{2 g} \alpha_{2 g+1}^{\prime}\right) \\
& \sim_{C \phi^{-2}}\left\{\alpha_{2 g+1}^{g+2} \cdot\left(\alpha_{2 g-1} \cdots \alpha_{3} \alpha_{1}\right) \cdot\left(\bar{\gamma}_{2 g} \cdots \bar{\gamma}_{4} \bar{\gamma}_{2}\right) \cdot\left(\beta_{2 g} \cdots \beta_{4} \beta_{2}\right)\right\} \\
& \cdot\left(\bar{\beta}_{2} \bar{\beta}_{4} \cdots \bar{\beta}_{2 g}\right) \cdot\left(\alpha_{1} \alpha_{2} \cdots \alpha_{2 g} \cdot \alpha_{2 g+1}^{g+1}\right) \cdot \alpha_{2 g+1}^{\prime} \\
& =\alpha_{2 g+1}^{g+2} \cdot\left(\alpha_{2 g-1} \cdots \alpha_{3} \alpha_{1}\right) \cdot{ }_{\phi^{-2}}\left\{\left(\bar{\gamma}_{2 g} \cdots \bar{\gamma}_{4} \bar{\gamma}_{2}\right) \cdot\left(\beta_{2 g} \cdots \beta_{4} \beta_{2}\right)\right\} \\
& \cdot\left(\bar{\beta}_{2} \bar{\beta}_{4} \cdots \bar{\beta}_{2 g}\right) \cdot\left(\alpha_{1} \alpha_{2} \cdots \alpha_{2 g} \cdot \alpha_{2 g+1}^{g+1}\right) \cdot \alpha_{2 g+1}^{\prime} \\
& \stackrel{(9)}{\longrightarrow} \alpha_{2 g+1}^{g+2} \cdot\left(\alpha_{2 g-1} \cdots \alpha_{3} \alpha_{1}\right) \cdot{ }_{\phi^{-2}}\left\{\left(\bar{\gamma}_{2 g} \cdots \bar{\gamma}_{4} \bar{\gamma}_{2}\right) \cdot\left(\beta_{2 g} \cdots \beta_{4} \beta_{2}\right)\right\} \\
& \cdot\left(\bar{\beta}_{2} \bar{\beta}_{4} \cdots \bar{\beta}_{2 g}\right) \cdot\left(\gamma_{2} \gamma_{4} \cdots \gamma_{2 g}\right) \cdot\left(\alpha_{1} \alpha_{3} \cdots \alpha_{2 g-1}\right) \cdot \alpha_{2 g+1}^{g+1} \cdot \alpha_{2 g+1}^{\prime} \\
& \stackrel{C}{\rightarrow}_{\phi^{-2}}\left\{\left(\bar{\gamma}_{2 g} \cdots \bar{\gamma}_{4} \bar{\gamma}_{2}\right) \cdot\left(\beta_{2 g} \cdots \beta_{4} \beta_{2}\right)\right\} \\
& \cdot\left(\bar{\beta}_{2} \bar{\beta}_{4} \cdots \bar{\beta}_{2 g}\right) \cdot\left(\gamma_{2} \gamma_{4} \cdots \gamma_{2 g}\right) \cdot \alpha_{1}^{2} \alpha_{3}^{2} \cdots \alpha_{2 g-1}^{2} \cdot \alpha_{2 g+1}^{2 g+3} \cdot \alpha_{2 g+1}^{\prime} \\
& \left.=\left({ }_{\phi^{-2}}\left(\bar{\gamma}_{2 g}\right) \cdots_{\phi^{-2}}\left(\bar{\gamma}_{4}\right) \cdot{ }_{\phi^{-2}}\left(\bar{\gamma}_{2}\right)\right) \cdot{ }_{\phi^{-2}}\left(\beta_{2 g}\right) \cdots_{\phi^{-2}}\left(\beta_{4}\right) \cdot{ }_{\phi^{-2}}\left(\beta_{2}\right)\right) \\
& \cdot\left(\bar{\beta}_{2} \bar{\beta}_{4} \cdots \bar{\beta}_{2 g}\right) \cdot\left(\gamma_{2} \gamma_{4} \cdots \gamma_{2 g}\right) \cdot \alpha_{1}^{2} \alpha_{3}^{2} \cdots \alpha_{2 g-1}^{2} \cdot \alpha_{2 g+1}^{2 g+3} \cdot \alpha_{2 g+1}^{\prime} \text {. }
\end{aligned}
$$

Note that, by Lemma 23, the operation $\stackrel{\phi^{-1}}{\longrightarrow}$ is also Hurwitz equivalent from [5, Lemma 6]. We have the following relation in $\Gamma_{g}^{8}$, which is Hurwitz equivalent to the relation $\delta=\left(\alpha_{2 g+1} \alpha_{2 g} \cdots \alpha_{2} \alpha_{1} \alpha_{1} \alpha_{2} \cdots \alpha_{2 g} \alpha_{2 g+1}^{\prime}\right)^{2}$ :

$$
\begin{aligned}
\delta= & \left({ }_{\phi^{-2}}\left(\bar{\gamma}_{2 g}\right) \cdots_{\phi^{-2}}\left(\bar{\gamma}_{4}\right) \cdot{ }_{\phi^{-2}}\left(\bar{\gamma}_{2}\right)\right) \cdot\left({ }_{\phi^{-2}}\left(\beta_{2 g}\right) \cdots{ }_{\phi^{-2}}\left(\beta_{4}\right) \cdot{ }_{\phi^{-2}}\left(\beta_{2}\right)\right) \\
& \cdot\left(\bar{\beta}_{2} \bar{\beta}_{4} \cdots \bar{\beta}_{2 g}\right) \cdot\left(\gamma_{2} \gamma_{4} \cdots \gamma_{2 g}\right) \cdot \alpha_{1}^{2} \alpha_{3}^{2} \cdots \alpha_{2 g-1}^{2} \cdot \alpha_{2 g+1}^{2 g+3} \cdot \alpha_{2 g+1}^{\prime} .
\end{aligned}
$$

By applying $\mathcal{D}_{g-1}$-substitutions twice to this relation, we have the following relation:

$$
\begin{aligned}
\delta= & \left({ }_{\phi^{-2}}\left(\bar{\gamma}_{2 g}\right) \cdots_{\phi^{-2}}\left(\bar{\gamma}_{4}\right) \cdot{ }_{\phi^{-2}}\left(\bar{\gamma}_{2}\right)\right) \cdot\left({ }_{\phi^{-2}}\left(\beta_{2 g}\right) \cdots{ }_{\phi^{-2}}\left(\beta_{4}\right) \cdot{ }_{\phi^{-2}}\left(\beta_{2}\right)\right) \\
& \cdot\left(\bar{\beta}_{2} \bar{\beta}_{4} \cdots \bar{\beta}_{2 g}\right) \cdot\left(\gamma_{2} \gamma_{4} \cdots \gamma_{2 g}\right) \cdot\left(\chi_{1} \chi_{2} \cdots \chi_{g}\right)^{2} \cdot \alpha_{2 g+1}^{7} \cdot \alpha_{2 g+1}^{\prime} .
\end{aligned}
$$

Moreover, by $\alpha_{2 g+1}^{7} \cdot \alpha_{2 g+1}^{\prime} \cdot \delta_{1} \delta_{2} \cdots \delta_{8}=\alpha_{2 g+1}^{7} \cdot \delta_{1} \delta_{2} \cdots \delta_{8} \cdot \alpha_{2 g+1}^{\prime}$ and the daisy relation $\alpha_{2 g+1}^{7} \cdot \delta_{1} \delta_{2} \cdots \delta_{8} \cdot \alpha_{2 g+1}^{\prime}=\zeta_{1} \zeta_{2} \cdots \zeta_{8} \cdot \delta$, we obtain

$$
\begin{aligned}
\delta \cdot \delta_{1} & \delta_{2} \cdots \delta_{8} \\
= & \left({ }_{\phi^{-2}}\left(\bar{\gamma}_{2 g}\right) \cdots{ }_{\phi^{-2}}\left(\bar{\gamma}_{4}\right) \cdot{ }_{\phi^{-2}}\left(\bar{\gamma}_{2}\right)\right) \cdot\left({ }_{\phi^{-2}}\left(\beta_{2 g}\right) \cdots_{\phi^{-2}}\left(\beta_{4}\right) \cdot{ }_{\phi^{-2}}\left(\beta_{2}\right)\right) \\
& \cdot\left(\bar{\beta}_{2} \bar{\beta}_{4} \cdots \bar{\beta}_{2 g}\right) \cdot\left(\gamma_{2} \gamma_{4} \cdots \gamma_{2 g}\right) \cdot\left(\chi_{1} \chi_{2} \cdots \chi_{g}\right)^{2} \\
& \cdot \alpha_{2 g+1}^{7} \cdot \alpha_{2 g+1}^{\prime} \cdot \delta_{1} \delta_{2} \cdots \delta_{8}
\end{aligned}
$$




$$
\begin{aligned}
= & \left({ }_{\phi^{-2}}\left(\bar{\gamma}_{2 g}\right) \cdots{ }_{\phi^{-2}}\left(\bar{\gamma}_{4}\right) \cdot{ }_{\phi^{-2}}\left(\bar{\gamma}_{2}\right)\right) \cdot\left({ }_{\phi^{-2}}\left(\beta_{2 g}\right) \cdots_{\phi^{-2}}\left(\beta_{4}\right) \cdot{ }_{\phi^{-2}}\left(\beta_{2}\right)\right) \\
& \cdot\left(\bar{\beta}_{2} \bar{\beta}_{4} \cdots \bar{\beta}_{2 g}\right) \cdot\left(\gamma_{2} \gamma_{4} \cdots \gamma_{2 g}\right) \cdot\left(\chi_{1} \chi_{2} \cdots \chi_{g}\right)^{2} \cdot \zeta_{1} \zeta_{2} \cdots \zeta_{8} \cdot \delta .
\end{aligned}
$$

Therefore, we have the following relation in $\Gamma_{g}^{8}$ :

$$
\begin{aligned}
\delta_{1} \delta_{2} \cdots \delta_{8}= & \left.{ }_{\phi^{-2}}\left(\bar{\gamma}_{2 g}\right) \cdots \phi_{\phi^{-2}}\left(\bar{\gamma}_{4}\right) \cdot{ }_{\phi^{-2}}\left(\bar{\gamma}_{2}\right)\right) \cdot\left({ }_{\phi^{-2}}\left(\beta_{2 g}\right) \cdots{ }_{\phi^{-2}}\left(\beta_{4}\right) \cdot{ }_{\phi^{-2}}\left(\beta_{2}\right)\right) \\
& \cdot\left(\bar{\beta}_{2} \bar{\beta}_{4} \cdots \bar{\beta}_{2 g}\right) \cdot\left(\gamma_{2} \gamma_{4} \cdots \gamma_{2 g}\right) \cdot\left(\chi_{1} \chi_{2} \cdots \chi_{g}\right)^{2} \cdot \zeta_{1} \zeta_{2} \cdots \zeta_{8} .
\end{aligned}
$$

It is easily seen that $\alpha_{i}$ and $\chi_{i}$ are mapped to $c_{i}$ and $x_{i}$, respectively, and $\zeta_{1}, \ldots, \zeta_{8}$ are mapped to $c_{2 g+1}$ under the map $\Gamma_{g}^{8} \rightarrow \Gamma_{g}$. This completes the proof.

For $j=1, \ldots, 2 g$, we write

$$
f_{j}=\left(c_{j-1} c_{j+1}\right)^{-1}\left(\bar{d}_{j}\right)
$$

where $c_{0}=1$.

\section{THEOREM 26}

Let $g \geq 3$. Then, the monodromy of the hyperelliptic Lefschetz fibration given by the word $H(g)=1$ can be conjugated to contain a daisy relations of type $2(g-1)$.

Proof

Since $\alpha_{i}$ and $\delta$ are mapped to $c_{i}$ and 1 under the map $\Gamma_{g}^{n} \rightarrow \Gamma_{g}$, respectively, by (12) acquired in Theorem 25, we obtain the following relator:

$$
\begin{aligned}
1= & \left({ }_{\varphi^{-2}}\left(\bar{e}_{2 g}\right) \cdots_{\varphi^{-2}}\left(\bar{e}_{4}\right) \cdot{ }_{\varphi^{-2}}\left(\bar{e}_{2}\right)\right) \cdot\left({ }_{\varphi^{-2}}\left(d_{2 g}\right) \cdots \varphi_{\varphi^{-2}}\left(d_{4}\right) \cdot{ }_{\varphi^{-2}}\left(d_{2}\right)\right) \\
& \cdot\left(\bar{d}_{2} \bar{d}_{4} \cdots \bar{d}_{2 g}\right) \cdot\left(e_{2} e_{4} \cdots e_{2 g}\right) \cdot c_{1}^{2} c_{3}^{2} \cdots c_{2 g-1}^{2} \cdot c_{2 g+1}^{2 g+4} .
\end{aligned}
$$

This relator contains the $D_{2(g-1)}$-relator. This completes the proof.

\section{THEOREM 27}

Let $g \geq 3$. Then, the monodromy of the Lefschetz fibration given by the word $I(g)=1$ can be conjugated to contain

(i) $g+1$ daisy relations of type $g-1$, and

(ii) $(g+1) / 2$ (resp., g/2) daisy relations of type $2(g-1)$ for odd (resp., even) $g$.

Proof

Let us first prove (i). Since $\varphi\left(c_{2 i-1}\right)=c_{2 i-1}$ for each $i=1, \ldots, g+1$, by Lemma 20(b) we have

$$
\begin{aligned}
\left(c_{1} c_{2} \cdots c_{2 g} c_{2 g+1}\right)^{2} & =\left(c_{1} c_{2} \cdots c_{2 g} c_{2 g+1}\right) \cdot\left(c_{1} c_{2} \cdots c_{2 g}\right) \cdot c_{2 g+1} \\
& \sim_{C}\left(\bar{d}_{2} \bar{d}_{4} \cdots \bar{d}_{2 g}\right) \cdot\left(c_{1} c_{2} \cdots c_{2 g} \cdot c_{2 g+1}^{g+1}\right) \cdot c_{2 g+1} .
\end{aligned}
$$


Moreover, we have

$$
c_{1} c_{2} c_{3} \cdots c_{2 g} \sim\left(e_{2} e_{4} e_{6} \cdots e_{2 g}\right) \cdot\left(c_{1} c_{3} c_{5} \cdots c_{2 g-1}\right) .
$$

Therefore, we have

$$
\begin{aligned}
\left(c_{1} c_{2} \cdots c_{2 g+1}\right)^{2 g+2} & \stackrel{(13)}{\longrightarrow}\left(\bar{d}_{2} \bar{d}_{4} \cdots \bar{d}_{2 g} \cdot c_{1} c_{2} \cdots c_{2 g} \cdot c_{2 g+1}^{g+2}\right)^{g+1} \\
& \stackrel{(14)}{\longrightarrow}\left(\bar{d}_{2} \bar{d}_{4} \cdots \bar{d}_{2 g} \cdot e_{2} e_{4} \cdots e_{2 g} \cdot c_{1} c_{3} \cdots c_{2 g-1} \cdot c_{2 g+1}^{g+2}\right)^{g+1} .
\end{aligned}
$$

Then, we see that we can apply $D_{g-1}^{\prime}$-substitution once and $D_{g-1}$-substitutions $g$ times to $\left(\bar{d}_{2} \bar{d}_{4} \cdots \bar{d}_{2 g} \cdot e_{2} e_{4} \cdots e_{2 g} \cdot c_{1} c_{3} \cdots c_{2 g-1} c_{2 g+1}^{g+2}\right)^{g+1}$. The reason that we apply $D_{g-1}^{\prime}$-substitution once is to construct a minimal symplectic manifold $Y(g, k)$ in Theorem 31 (see the proof of Theorem 31 and Remark 36). This completes the proof of (i).

Next we prove (ii). By Lemma 19(b) we have

$$
\begin{aligned}
\left(c_{1} c_{2} \cdots c_{2 g} c_{2 g+1}\right)^{2} & =\left(c_{1} c_{2} \cdots c_{2 g} c_{2 g+1}\right) \cdot\left(c_{1} c_{2} \cdots c_{2 g}\right) \cdot c_{2 g+1} \\
& \sim\left(d_{1} d_{2} \cdots d_{2 g} \cdot c_{2 g+1}^{2 g+1}\right) \cdot c_{2 g+1} .
\end{aligned}
$$

Moreover, we have

$$
\left(c_{1} c_{2} \cdots c_{2 g+1}\right)^{2} \sim\left(c_{1}^{2} c_{3}^{2} \cdots c_{2 g+1}^{2}\right) \cdot\left(f_{2} f_{4} \cdots f_{2 g}\right) \cdot\left(\bar{d}_{2} \bar{d}_{4} \cdots \bar{d}_{2 g}\right) .
$$

From the above relations, we have

$$
\begin{aligned}
& \left(c_{1} c_{2} \cdots c_{2 g} c_{2 g+1}\right)^{4} \\
& \quad \stackrel{(15)}{\longrightarrow}\left(d_{1} d_{2} \cdots d_{2 g} \cdot c_{2 g+1}^{2 g+2}\right) \cdot\left(c_{1} c_{2} \cdots c_{2 g} c_{2 g+1}\right)^{2} \\
& \quad \stackrel{(16)}{\longrightarrow}\left(d_{1} d_{2} \cdots d_{2 g} \cdot c_{2 g+1}^{2 g+2}\right) \cdot\left(c_{1}^{2} c_{3}^{2} \cdots c_{2 g+1}^{2}\right) \cdot\left(f_{2} f_{4} \cdots f_{2 g}\right) \cdot\left(\bar{d}_{2} \bar{d}_{4} \cdots \bar{d}_{2 g}\right) \\
& \quad \sim\left(d_{1} d_{2} \cdots d_{2 g}\right) \cdot\left(c_{1}^{2} c_{3}^{2} \cdots c_{2 g-1}^{2} c_{2 g+1}^{2 g+4}\right) \cdot\left(f_{2} f_{4} \cdots f_{2 g}\right) \cdot\left(\bar{d}_{2} \bar{d}_{4} \cdots \bar{d}_{2 g}\right) .
\end{aligned}
$$

From this, we see that $\left(c_{1} c_{2} \cdots c_{2 g} c_{2 g+1}\right)^{4}$ can be conjugated to contain daisy relations of type $2(g-1)$. Therefore,

$$
I(g)= \begin{cases}\left(c_{1} c_{2} \cdots c_{2 g+1}\right)^{4 k} \cdot\left(c_{1} c_{2} \cdots c_{2 g+1}\right)^{2} & g=2 k, \\ \left(c_{1} c_{2} \cdots c_{2 g+1}\right)^{4(k+1)} & g=2 k+1\end{cases}
$$

gives the proof of (ii).

\section{THEOREM 28}

Let $g \geq 3$. Then, the monodromy of the Lefschetz fibration given by the word $G(g)=1$ can be conjugated to contain $g$ daisy relations of type $2(g-1)$.

\section{Proof}

By a similar argument to the proof of Theorem 27, we have

$$
\begin{aligned}
& \left(c_{1} \cdots c_{2 g-1} c_{2 g}\right)^{4} \\
& \quad \sim\left(d_{1} d_{2} \cdots d_{2 g-1}\right) \cdot\left(c_{2}^{2} c_{4}^{2} \cdots c_{2 g-2}^{2} c_{2 g}^{2 g+3}\right) \cdot\left(f_{1} f_{3} \cdots f_{2 g-1}\right) \cdot\left(\bar{d}_{1} \bar{d}_{3} \cdots \bar{d}_{2 g-1}\right) .
\end{aligned}
$$


Let $h:=\left(a_{1} \cdots a_{2 g} a_{2 g+1}\right)$. Note that $h\left(a_{i}\right)=a_{i+1}$ for $i=1, \ldots, 2 g$. Then, we have

$$
\begin{aligned}
& \left(c_{1} \cdots c_{2 g-1} c_{2 g}\right)^{4} \\
& \quad \sim\left(d_{1} d_{2} \cdots d_{2 g-1}\right) \cdot\left(c_{2}^{2} c_{4}^{2} \cdots c_{2 g-2}^{2} c_{2 g}^{2 g+3}\right) \cdot\left(f_{1} f_{3} \cdots f_{2 g-1}\right) \cdot\left(\bar{d}_{1} \bar{d}_{3} \cdots \bar{d}_{2 g-1}\right) \\
& \quad \stackrel{h}{\rightarrow}\left(d_{2} d_{3} \cdots d_{2 g}\right) \cdot\left(c_{3}^{2} c_{5}^{2} \cdots c_{2 g-1}^{2} c_{2 g+1}^{2 g+3}\right) \cdot\left(f_{2} f_{3} \cdots f_{2 g}\right) \cdot\left(\bar{d}_{2} \bar{d}_{4} \cdots \bar{d}_{2 g}\right) .
\end{aligned}
$$

From this, we see that $\left(c_{1} c_{2} \cdots c_{2 g} c_{2 g}\right)^{4}$ can be conjugated to contain daisy relations of type $2(g-1)$. Then $G(g)=\left(c_{1} \cdots c_{2 g-1} c_{2 g}\right)^{4 g} \cdot\left(c_{1} \cdots c_{2 g-1} c_{2 g}\right)^{2}$ gives the proof.

\subsection{Nonhyperellipticity of our Lefschetz fibrations}

The purpose of this section is to prove that all the Lefschetz fibrations obtained in this article via daisy substitutions are nonhyperelliptic. The proof will be obtained as a corollary of the more general theorem given below.

\section{THEOREM 29}

Let $g \geq 3$. Let $f_{\varrho_{1}}: X_{\varrho_{1}} \rightarrow \mathbb{S}^{2}$ be a genus $g$ hyperelliptic Lefschetz fibration with only nonseparating vanishing cycles, and let $\varrho_{1}$ be a positive relator corresponding to $f$. Let $k_{1}$ and $k_{2}$ be nonnegative integers such that $k_{1}+k_{2}>0$, and let $k$ be a positive integer. Then we have the following.

(i) If we can obtain a positive relator, denoted by $\varrho_{2}$, by applying $D_{g-1^{-}}$ substitutions $k_{1}$ times and $D_{g-1}^{\prime}$-substitutions $k_{2}$ times to $\varrho$, then the genus $g$ Lefschetz fibration $f_{\varrho_{2}}: X_{\varrho_{2}} \rightarrow \mathbb{S}^{2}$ is nonhyperelliptic.

(ii) If we can obtain a positive relator, denoted by $\varrho_{3}$, by applying $D_{2(g-1)^{-}}$ substitutions $k$ times to $\varrho$, then the genus $g$ Lefschetz fibration $f_{\varrho_{3}}: X_{\varrho_{3}} \rightarrow \mathbb{S}^{2}$ is nonhyperelliptic.

\section{COROLLARY 30}

All our Lefschetz fibrations are nonhyperelliptic.

\section{Proof of Theorem 29}

Let $s_{0}$ be the number of nonseparating vanishing cycles of $f_{\varrho_{1}}$. Note that, by Theorem 7, we have $\sigma\left(X_{\varrho_{1}}\right)=-\frac{g+1}{2 g+1} s_{0}$.

First, we assume that $f_{\varrho_{2}}$ is a hyperelliptic Lefschetz fibration. The relators $D_{g-1}$ and $D_{g-1}^{\prime}$ consist of only Dehn twists about nonseparating simple closed curves $c_{1}, c_{3}, \ldots, c_{2 g+1}$ and $x_{1}, x_{1}^{\prime}, \ldots, x_{g}, x_{g}^{\prime}$ as in Figure 5. Therefore, we see that $\varrho_{2}$ consists of only right-handed Dehn twists about nonseparating simple closed curves, so $f_{\varrho_{2}}$ has only nonseparating vanishing cycles. In particular, the number of nonseparating vanishing cycles of $f_{\varrho_{2}}$ is $s_{0}-\{(g-1)-1\}\left(k_{1}+k_{2}\right)$. By Theorem 7, we have

$$
\sigma\left(X_{\varrho_{2}}\right)=-\frac{g+1}{2 g+1}\left\{s_{0}-(g-2)\left(k_{1}+k_{2}\right)\right\} .
$$


On the other hand, since the relators $D_{g-1}$ and $D_{g-1}^{\prime}$ are daisy relators of type $g-1$, by Theorem 17, we have

$$
\sigma\left(X_{\varrho_{2}}\right)=\sigma\left(X_{\varrho_{1}}\right)+(g-2)\left(k_{1}+k_{2}\right)=-\frac{g+1}{2 g+1} s_{0}+(g-2)\left(k_{1}+k_{2}\right) .
$$

We get a contradiction since the above equality does not hold for $g \geq 3$ and $k_{1}+k_{2}>0$.

Next, we assume that $f_{\varrho_{3}}$ is a hyperelliptic Lefschetz fibration. The relator $D_{2(g-1)}$ consists of Dehn twists about nonseparating simple closed curves $c_{3}, c_{5}, \ldots, c_{2 g+1}$ and $y_{2}, y_{3}, \ldots, y_{2 g-1}$ in Figure 5 and a Dehn twist about the separating simple closed curve $y_{1}$ in Figure 5 . Note that $y_{g+1}=x_{2}, y_{g+2}=x_{3}, \ldots$, $y_{2 g-1}=x_{g}$, and note that $y_{1}$ separates $\Sigma_{g}$ into two surfaces, one of which has genus 1 . Therefore, $f_{\varrho_{3}}$ has $s_{0}-k\{2(g-1)\}$ nonseparating vanishing cycles and $k$ separating vanishing cycles which are $y_{1}$. By Theorem 7 , we have

$$
\begin{aligned}
\sigma\left(X_{\varrho_{3}}\right) & =-\frac{g+1}{2 g+1}\left\{s_{0}-2 k(g-1)\right\}+\left(\frac{4(g-1)}{2 g+1}-1\right) k \\
& =-\frac{g+1}{2 g+1} s_{0}+\frac{2 g^{2}+2 g-7}{2 g+1} k .
\end{aligned}
$$

On the other hand, since the relator $D_{2(g-1)}$ is a daisy relator of type $2(g-1)$, by Theorem 17, we have

$$
\sigma\left(X_{\varrho_{3}}\right)=\sigma\left(X_{\varrho_{1}}\right)+(2 g-3) k=-\frac{g+1}{2 g+1} s_{0}+(2 g-3) k .
$$

Since $g \geq 3$, we have

$$
\begin{aligned}
& \left(-\frac{g+1}{2 g+1} s_{0}+(2 g-3) k\right)-\left(-\frac{g+1}{2 g+1} s_{0}+\frac{2 g^{2}+2 g-7}{2 g+1} k\right) \\
& \quad=\frac{2(g-1)(g-2)}{2 g+1} k>0 .
\end{aligned}
$$

This is a contradiction to the above equality.

\section{Constructing exotic 4-manifolds}

The purpose of this section is to show that the symplectic 4-manifolds obtained in Theorem 27(i) are irreducible. Moreover, by performing the knot surgery operation along a homologically essential torus on these symplectic 4-manifolds, we obtain infinite families of mutually nondiffeomorphic irreducible smooth structures.

\section{THEOREM 31}

Let $g \geq 3$, and let $M$ be one of the following 4-manifolds: $\left(g^{2}-g+1\right) \mathbb{C P}^{2} \#\left(3 g^{2}+\right.$ $3 g+3-(g-2) k) \overline{\mathbb{C P}}^{2}$ for $k=2, \ldots, g+1$. There exists an irreducible symplectic 4-manifold $Y(g, k)$ homeomorphic but not diffeomorphic to $M$ that can be 
obtained from the genus $g$ Lefschetz fibration on $Y(g)$ over $\mathbb{S}^{2}$ with the monodromy $\left(c_{1} c_{2} \cdots c_{2 g+1}\right)^{2 g+2}=1$ in the mapping class group $\Gamma_{g}$ by applying $k$ daisy substitutions of type $g-1$.

Proof

Let $Y(g, k)$ denote the symplectic 4-manifold obtained from $Y(g)=W(g) \# 2 \overline{\mathbb{C P}}^{2}$ by applying $k-1 D_{g-1}$-substitutions and one $D_{g-1}^{\prime}$-substitution as in Theorem 27. Applying Lemma 14, we compute the topological invariants of $Y(g, k)$ as

$$
\begin{aligned}
e(Y(g, k)) & =e\left(W(g) \# 2 \overline{\mathbb{C P}}^{2}\right)-k(g-2)=2\left(2 g^{2}+g+3\right)-k(g-2), \\
\sigma(Y(g, k)) & =\sigma\left(W(g) \# 2 \overline{\mathbb{C P}}^{2}\right)+k(g-2)=-2(g+1)^{2}+k(g-2) .
\end{aligned}
$$

Using the factorization of the global monodromy in terms of right-handed Dehn twists of the genus $g$ Lefschetz fibration on $Y(g, k)$ (see Theorem 27(i)), it is easy to check that $\pi_{1}(Y(g, k))=1$.

Next, we show that $Y(g, k)$ is a nonspin 4 -manifold. Let $c_{1}, \ldots, c_{2 g+1}$ be the curves in Figure 2, and let $x_{1}, \ldots, x_{g}, x_{1}^{\prime}, \ldots, x_{g}^{\prime}$ be the curves in Figure 5. Note that $\bar{d}_{i}={ }_{c_{i+1}^{-1}}\left(c_{i}\right)$ and $e_{i+1}={ }_{c_{i}}\left(c_{i+1}\right)$. The vanishing cycles of Lefschetz fibrations in Theorem 27 include the curves $\bar{d}_{2 i}, e_{2 i}$ for $i=1, \ldots, g, x_{j}, x_{j}^{\prime}$ for $j=1, \ldots, g$, and $c_{2 g+1}$. In $H_{1}\left(\Sigma_{g} ; \mathbb{Z}_{2}\right)$, we find that $\bar{d}_{2 g}=c_{2 g+1}+c_{2 g}, e_{2 g}=$ $c_{2 g-1}+c_{2 g}$, and $x_{g}=c_{2 g-1}+c_{2 g+1}$. Therefore, we have $\bar{d}_{2 g}+e_{2 g}=x_{g}$. In the notation of Theorem 8 , we have $l=2$ and $\bar{d}_{2 g} \cdot e_{2 g}=0$. Therefore, $2+\bar{d}_{2 g} \cdot e_{2 g} \equiv 0$ $(\bmod 2)$.

By Theorem 9, $Y(g, k)$ is nonspin, and thus, it has an odd intersection form. By Freedman's classification (see [16]), we see that $Y(g, k)$ is homeomorphic to $\left(g^{2}-g+1\right) \mathbb{C P}^{2} \#\left(3 g^{2}+3 g+3-(g-2) k\right) \overline{\mathbb{C P}}^{2}$.

Next, using the fact that $W(g)$ is a minimal complex surface of general type with $b_{2}{ }^{+}>1$ and the blow-up formula for the Seiberg-Witten function (see [15]), we compute $S W_{W(g) \# 2 \overline{\mathbb{C P}}^{2}}=S W_{W(g)} \cdot \prod_{j=1}^{2}\left(e^{E_{i}}+e^{-E_{i}}\right)=\left(e^{K_{W(g)}}+\right.$ $\left.e^{-K_{W(g)}}\right)\left(e^{E_{1}}+e^{-E_{1}}\right)\left(e^{E_{2}}+e^{-E_{2}}\right)$, where $E_{i}$ denotes the exceptional class of the $i$ th blowup. By the above formula, the SW basic classes of $W(g) \# 2 \overline{\mathbb{C P}}^{2}$ are given by $\pm K_{W(g)} \pm E_{1} \pm E_{2}$, and the values of the Seiberg-Witten invariants on these classes are \pm 1 . Notice that, by [14, Corollary 8.6], $Y(g, k)$ has Seiberg-Witten simple type. Furthermore, by applying Theorems 15 and 16, we completely determine the Seiberg-Witten invariants of $Y(g, k)$ by using the basic classes and invariants of $W(g) \# 2 \overline{\mathbb{C P}}^{2}$. Up to sign, the symplectic manifold $Y(g, k)$ has only one basic class which descends from the \pm canonical class of $Y(g)$ (see the detailed explanation below). By Theorem 16 or Taubes's work [36], the values of the Seiberg-Witten function on these basic classes, $\pm K_{Y(g, k)}$, are \pm 1 .

In what follows, we spell out the details of the above discussion. By Theorems 15 and 16, we can determine the Seiberg-Witten invariants of $Y(g, k)$ by computing the algebraic intersection number of the basic classes $\pm K_{W(g)} \pm E_{1} \pm E_{2}$ of $W(g) \# 2 \overline{\mathbb{C P}}^{2}$, with the classes of spheres of $k$ disjoint $C_{g-1}$ configurations in $Y(g)$. Notice that the leading spheres of the configurations $C_{g-1}$ are the components 
of the singular fibers of $Y(g)$. By looking at the regions on the genus $g$ surface, where the rational blowdowns along $C_{g-1}$ are performed, and the location of the base points of the genus $g$ pencil, we compute the algebraic intersection numbers as follows. Let $S_{1}^{j}$ denote the homology class of the $-(g+1)$-sphere of the $j$ th configurations $C_{g-1}$, and let $S_{2}^{j}, \ldots, S_{g-2}^{j}$ be the homology classes of $(-2)$ spheres of $C_{g-1}$ in $W(g) \# 2 \overline{\mathbb{C P}}^{2}$, where $j=1,2$. These two rational blowdowns along $C_{g-1}$ are chosen such that they correspond to one $D_{g-1}$-substitution and one $D_{g-1}^{\prime}$-substitution as in Theorem 27(i).

We have $S_{1}^{1} \cdot E_{1}=1, S_{1}^{1} \cdot E_{2}=0, S_{1}^{2} \cdot E_{2}=1, S_{1}^{2} \cdot E_{1}=0, S_{1}^{j} \cdot K_{W g}=g-2$, and the canonical divisor does not intersect with $S_{i}^{j}$ for $2 \leq i \leq g-1$. Consequently, $S_{1}^{j} \cdot \pm\left(K_{W g}+E_{1}+E_{2}\right)= \pm(g-1)$ for $j=1,2$, and $S_{1}^{j} \cdot\left( \pm K_{W g} \pm E_{1} \mp E_{2}\right) \neq$ $\pm(g-1)$ for one $j$. Observe that among the eight basic classes $\pm K_{W g} \pm E_{1} \pm E_{2}$, only $K_{W g}+E_{1}+E_{2}$ and $-\left(K_{W g}+E_{1}+E_{2}\right)$ have algebraic intersection $\pm(g-1)$ with $-(g+1)$-spheres of $C_{g-1}$. Thus, Theorem 15 implies that these are the only two basic classes that descend to $Y(g, 2)$ and, consequently, to $Y(g, k)$ from $W(g) \# 2 \overline{\mathbb{C P}}^{2}$.

By invoking the connected sum theorem for Seiberg-Witten invariants, we see that the $S W$ function is trivial for $\left(g^{2}-g+1\right) \mathbb{C P}^{2} \#\left(3 g^{2}+3 g+3-\right.$ $(g-2) k) \overline{\mathbb{C P}}^{2}$. Since the Seiberg-Witten invariants are diffeomorphism invariants, $Y(g, k)$ is not diffeomorphic to $\left(g^{2}-g+1\right) \mathbb{C P}^{2} \#\left(3 g^{2}+3 g+3-(g-2) k\right) \overline{\mathbb{C P}}^{2}$.

The minimality of $Y(g, k)$ is a consequence of the fact that $Y(g, k)$ has no two Seiberg-Witten basic classes $K$ and $K^{\prime}$ such that $\left(K-K^{\prime}\right)^{2}=-4$. Notice that $\pm K_{Y(g, k)}$ are the only basic classes of $Y(g, k)$, and note that $\left(K_{Y(g, k)}^{2}-\right.$ $\left.\left(-K_{Y(g, k)}\right)\right)^{2}=4\left(K_{Y(g, k)}^{2}\right) \geq 0$. Thus, we conclude that $Y(g, k)$ is symplectically minimal. Furthermore, since symplectic minimality implies irreducibility for simply connected 4-manifolds (see [21]), we deduce that $Y(g, k)$ is also smoothly irreducible.

The analogous for $g=2$, using lantern substitution, was proved in [3] (see Theorem 17).

\section{THEOREM 32}

There exists an infinite family of irreducible symplectic and an infinite family of irreducible nonsymplectic pairwise nondiffeomorphic 4-manifolds all homeomorphic to $Y(g, k)$.

\section{Proof}

We have that $Y(g, k)$ contains $g(g-1)$ Lagrangian tori which are disjoint from the singular fibers of the genus $g$ Lefschetz fibration on $Y(g, k)$. These tori descend from $W(g)$ (see Example 12) and survive in $Y(g, k)$ after the rational blowdowns along $C_{g-1}$. These tori are Lagrangian, but we can perturb the symplectic form on $Y(g, k)$ so that one of these tori, say, $T$, becomes symplectic. Moreover, $\pi_{1}(Y(g, k) \backslash T)=1$, which follows from Van Kampen's theorem by using the facts that $\pi_{1}(Y(g, k))=1$ and any rim torus has a dual $(-2)$-sphere (see [20, 
Proposition 1.2] or Gompf [18, p. 564]). Hence, we have a symplectic torus $T$ in $Y(g, k)$ of self-intersection 0 such that $\pi_{1}(Y(g, k) \backslash T)=1$. By performing a knot surgery on $T$, inside $Y(g, k)$, we obtain an irreducible 4-manifold $Y(g, k)_{K}$ that is homeomorphic to $Y(g, k)$. By varying our choice of the knot $K$, we can realize infinitely many pairwise nondiffeomorphic 4-manifolds, either symplectic or nonsymplectic.

\section{REMARK 33}

We obtain analogous results to Theorem 31 for the genus $g$ Lefschetz fibrations obtained in Theorem 28. The total space $Z(g)$ of the Lefschetz fibration with the monodromy $\left(c_{1} c_{2} \cdots c_{2 g+1}\right)^{2(2 g+1)}=1$ in the mapping class group $\Gamma_{g}$ is the complex surface of general type with $b_{2}{ }^{+}>1$ and the single blowup of a minimal complex surface (see [19, Section 8.4, pp. 320-322]). The computation of the Seiberg-Witten invariants follows the same line of argument as that of Theorem 31. In fact, the SW computation is simpler, since $Z(g)$ only admits one pair of basic classes. Also, the results of our article can be easily extendable to the Lefschetz fibrations with monodromies $\left(c_{1} c_{2} \cdots c_{2 g-1} c_{2 g} c_{2 g+1}^{2} c_{2 g} c_{2 g-1} \cdots c_{2} c_{1}\right)^{2 n}=$ $1,\left(c_{1} c_{2} \cdots c_{2 g} c_{2 g+1}\right)^{(2 g+2) n}=1$, and $\left(c_{1} c_{2} \cdots c_{2 g-1} c_{2 g}\right)^{2(2 g+1) n}=1$ for $n \geq 2$. Since the computations are lengthy, we will not present them here.

\section{REMARK 34}

Note that all the Lefschetz fibrations constructed in our article are nonspin. The fibrations obtained in Theorems 24 and 25 admit a section of self-intersection -1 . The fibrations in Theorem 26 and 28 contain separating vanishing cycles. (The curve $y_{1}$ in Figure 5 is separating.) Thus, the total spaces are nonspin. In general, if we apply the daisy substitution of type $2(g-1)$ to a positive relator in $\Gamma_{g}$, then the resulting Lefschetz fibration always contains separating vanishing cycles. The fibrations in Theorem 27 do not contain any separating vanishing cycles, but they are nonspin due to Stipsicz's criteria (see proof of Theorem 31).

\section{REMARK 35}

It would be interesting to know if an analogue of Theorem 31 holds for the Lefschetz fibrations of Theorem 25. Their corresponding monodromies are obtained by applying $D_{g-1}$-substitutions twice to $H(g)$. In the opposite direction, we can prove that the total spaces of the Lefschetz fibrations of Theorem 24, whose monodromies are obtained by applying one $D_{g-1}$-substitution to $H(g)$, are blowups of the complex projective plane. Notice that by Theorem 24 they admit at least $(2 g+6)$-sphere sections of self-intersection -1 . By using a result of Y. Sato [32, Theorem 1.2, p. 194], we see that the total spaces of these Lefschetz fibrations are diffeomorphic to $\mathbb{C P}^{2} \#(3 g+5) \overline{\mathbb{C P}}^{2}$.

\section{REMARK 36}

In the proof of Theorem 27(i), if we apply $k D_{g-1}$-substitutions for $k=1, \ldots, g+1$ without applying a $D_{g-1}^{\prime}$-substitution, then the Lefschetz fibrations over $\mathbb{S}^{2}$ given 
by the resulting relations admit a section of self-intersection -1 . (That is, the total spaces of the fibrations are nonminimal.)

Acknowledgments. The authors are grateful to the referee for valuable comments and suggestions.

\section{References}

[1] S. Akbulut and B. Ozbagci, On the topology of compact Stein surfaces, Int. Math. Res. Not. IMRN 2002, no. 15, 769-782. MR 1891172.

DOI $10.1155 /$ S1073792802108105.

[2] A. Akhmedov and B. Ozbagci, Exotic Stein fillings with arbitrary fundamental group, preprint, arXiv:1212.1743v2 [math.GT].

[3] A. Akhmedov and J.-Y. Park, Lantern substitution and new symplectic 4-manifolds with $b_{2}^{+}=3$, Math. Res. Lett. 21 (2014), 1-17. MR 3247035. DOI 10.4310/MRL.2014.v21.n1.a1.

[4] D. Auroux, Fiber sums of genus 2 Lefschetz fibrations, Turkish J. Math. 27 (2003), 1-10. MR 1975329.

[5] - A stable classification of Lefschetz fibrations, Geom. Topol. 9 (2005), 203-217. MR 2115673. DOI 10.2140/gt.2005.9.203.

[6] D. Auroux and L. Katzarkov, Branched coverings of $\mathbb{C P}^{2}$ and invariants of symplectic 4-manifolds, Invent. Math. 142 (2000), 631-673. MR 1804164. DOI 10.1007/PL00005795.

[7] R. İ. Baykur, M. Korkmaz, and N. Monden, Sections of surface bundles and Lefschetz fibrations, Trans. Amer. Math. Soc. 365 (2013), no. 11, 5999-6016. MR 3091273. DOI 10.1090/S0002-9947-2013-05840-0.

[8] M. Dehn, Die Gruppe der Abbildungsklassen, Acta Math. 69 (1938), 135-206. MR 1555438. DOI 10.1007/BF02547712.

[9] S. K. Donaldson, Lefschetz pencils on symplectic manifolds, J. Differential Geom. 53 (1999), 205-236. MR 1802722.

[10] H. Endo, Meyer's signature cocycle and hyperelliptic fibrations, Math. Ann. 316 (2000), 237-257. MR 1741270. DOI 10.1007/s002080050012.

[11] H. Endo and Y. Z. Gurtas, Lantern relations and rational blowdowns, Proc. Amer. Math. Soc. 138 (2010), 1131-1142. MR 2566578. DOI 10.1090/S0002-9939-09-10128-4.

[12] H. Endo, T. E. Mark, and J. Van Horn-Morris, Monodromy substitutions and rational blowdowns, J. Topol. 4 (2011), 227-253. MR 2783383. DOI 10.1112/jtopol/jtq041.

[13] H. Endo and S. Nagami, Signature of relations in mapping class groups and non-holomorphic Lefschetz fibrations, Trans. Amer. Math. Soc. 357 (2005), no. 8, 3179-3199. MR 2135741. DOI 10.1090/S0002-9947-04-03643-8. 
[14] R. Fintushel and R. J. Stern, Rational blowdowns of smooth 4-manifolds, J. Differential Geom. 46 (1997), 181-235. MR 1484044.

[15] Knots, links, and 4-manifolds, Invent. Math. 134 (1998), 363-400. MR 1650308. DOI 10.1007/s002220050268.

[16] M. H. Freedman, The topology of four-dimensional manifolds, J. Differential Geom. 17 (1982), 357-453. MR 0679066.

[17] T. Fuller, Generalized nuclei of complex surfaces, Pacific. J. Math. 187 (1999), 281-295. MR 1675025. DOI 10.2140/pjm.1999.187.281.

[18] R. E. Gompf, A new construction of symplectic manifolds, Ann. of Math. (2) 142 (1995), 527-595. MR 1356781. DOI 10.2307/2118554.

[19] R. E. Gompf and A. I. Stipsicz, 4-Manifolds and Kirby Calculus, Grad. Stud. Math. 20, Amer. Math. Soc., Providence, 1999. MR 1707327.

DOI $10.1090 / \mathrm{gsm} / 020$.

[20] M. Halic, On the geography of symplectic 6-manifolds, Manuscripta Math. 99 (1999), 371-381. MR 1702585. DOI 10.1007/s002290050179.

[21] M. J. D. Hamilton and D. Kotschick, Minimality and irreducibility of symplectic four-manifolds, Int. Math. Res. Not. IMRN 2006, art. ID 35032. MR 2211144. DOI 10.1155/IMRN/2006/35032.

[22] N. V. Ivanov, "Mapping class groups" in Handbook of Geometric Topology, North-Holland, Amsterdam, 2002, 523-633. MR 1886678.

[23] D. L. Johnson, Homeomorphisms of a surface which act trivially on homology, Proc. Amer. Math. Soc. 75 (1979), 119-125. MR 0529227. DOI $10.2307 / 2042686$.

[24] A. Kas, On the handlebody decomposition associated to a Lefschetz fibration, Pacific J. Math. 89 (1980), 89-104. MR 0596919.

[25] M. Korkmaz and B. Ozbagci, On sections of elliptic fibrations, Michigan Math. J. 56 (2008), 77-87. MR 2433657. DOI 10.1307/mmj/1213972398.

[26] Y. Matsumoto, On 4-manifolds fibered by tori, II, Proc. Japan Acad. Ser. A Math. Sci. 59 (1983), 100-103. MR 0711307.

[27] - "Lefschetz fibrations of genus two - a topological approach" in Topology and Teichmüller Spaces (Katinkulta, 1995), World Sci., River Edge, N.J., 1996, 123-148. MR 1659687.

[28] S. C.. Onaran, On sections of genus two Lefschetz fibrations, Pacific J. Math. 248 (2010), 203-216. MR 2734172. DOI 10.2140/pjm.2010.248.203.

[29] J. Park, Seiberg-Witten invariants of generalised rational blow-downs, Bull. Austral. Math. Soc. 56 (1997), 363-384. MR 1490654. DOI $10.1017 /$ S0004972700031154.

[30] O. Plamenevskaya and J. Van Horn-Morris, Planar open books, monodromy factorizations and symplectic fillings, Geom. Topol. 14 (2010), 2077-2101. MR 2740642. DOI 10.2140/gt.2010.14.2077.

[31] V. A. Rohlin, New results in the theory of four-dimensional manifolds, Doklady Akad. Nauk SSSR (N.S.) 84 (1952), 221-224. MR 0052101. 
[32] Y. Sato, Canonical classes and the geography of nonminimal Lefschetz fibrations over $S^{2}$, Pacific J. Math. 262 (2013), 191-226. MR 3069059.

DOI 10.2140/pjm.2013.262.191.

[33] I. Smith, Geometric monodromy and the hyperbolic disc, Q. J. Math. 52 (2001), 217-228. MR 1838364. DOI 10.1093/qjmath/52.2.217.

[34] A. I. Stipsicz, Spin structures on Lefschetz fibrations, Bull. Lond. Math. Soc. 33 (2001), 466-472. MR 1832559. DOI 10.1017/S0024609301008232.

[35] S. Tanaka, On sections of hyperelliptic Lefschetz fibrations, Algebr. Geom. Topol. 12 (2012), 2259-2286. MR 3020206. DOI 10.2140/agt.2012.12.2259.

[36] C. H. Taubes, The Seiberg-Witten invariants and symplectic forms, Math. Res. Lett. 1 (1994), 809-822. MR 1306023. DOI 10.4310/MRL.1994.v1.n6.a15.

[37] M. Teicher, "Braid groups, algebraic surfaces and fundamental groups of complements of branch curves" in Algebraic Geometry - Santa Cruz 1995, Proc. Sympos. Pure Math. 62, Amer. Math. Soc., Providence, 1997, 127-150. MR 1492521.

Akhmedov: School of Mathematics, University of Minnesota, Minneapolis, Minnesota, USA; akhmedov@math.umn.edu

Monden: Department of Engineering Science, Osaka Electro-Communication University, Neyagawa, Japan; monden@isc.osakac.ac.jp 